\title{
Les îles au bout du temps
}

Essai chronométrique sur les Philippines espagnoles $\mathrm{XVI}^{\mathrm{e}}-\mathrm{XVII}{ }^{\mathrm{e}}$ siècles

The Islands at the End of Time. A Chronometric Essay on the Spanish Philippines $16^{\text {th }}-17^{\text {th }}$ Centuries

Jean-Noël Sanchez

\section{OpenEdition \\ Journals}

Édition électronique

URL : https://journals.openedition.org/cher/4229

DOI : $10.4000 /$ cher.4229

ISSN : 2803-5992

Éditeur

Presses universitaires de Strasbourg

Édition imprimée

Date de publication : 30 novembre 2021

Pagination : 47-79

ISBN : 979-10-344-0095-9

ISSN : 1968-035X

\section{Référence électronique}

Jean-Noël Sanchez, «Les îles au bout du temps », reCHERches [En ligne], 27 | 2021, mis en ligne le 30 novembre 2021, consulté le 12 janvier 2022. URL : http://journals.openedition.org/cher/4229 ; DOI : https://doi.org/10.4000/cher.4229

\section{cc) (†) (-)}

Ce(tte) œuvre est mise à disposition selon les termes de la Licence Creative Commons Attribution -

Pas d'Utilisation Commerciale - Partage dans les Mêmes Conditions 4.0 International. 


\title{
Les îles au bout du temps Essai chronométrique sur les Philippines espagnoles XVIe-XVIIe siècles
}

\author{
JeAN-Nö̈L SANCHEZ*
}

Le temps s'en va, le temps s'en va, madame; Las! Le temps, non, mais nous nous en allons.

Pierre de Ronsard, Continuation des amours

\begin{abstract}
T 'Histoire aime à se donner un début, un premier jalon qui porterait en Llui, déjà, un indice, une clé, une «symptomaticité» de la période qu'en conséquence il inaugure.

Ainsi, l'année 1492, non pas tant pour la chute le 7 novembre d'une prodigieuse météorite de 127 kilogrammes à Ensisheim, en Alsace (Bigot Morogues 1812: 58), que pour l'arrivée le mois précédent d'un Génois sur la plage d'un îlot dont on ne déterminera sans doute jamais la localisation exacte, marqua assurément le début d'une nouvelle ère, et fut d'ailleurs parfois proposée comme un autre point de départ de la Renaissance, si l'on entend celle-ci comme le vestibule de cette «Europa portátil», selon la lumineuse expression de Baltasar Gracián (1651), qui braverait les océans pour triompher du monde.
\end{abstract}

Cependant, si ce sont aux Indes de Castille auxquelles nous tâchons de donner un début, l'excursion colombine n'est peut-être pas le prisme nous donnant le mieux à voir ce que deviendra l'Amérique hispanique. 1501, année de rédaction des instructions données à Nicolas de Ovando, futur premier gouverneur d'Hispaniola, dans lesquelles le terme d'Indio n'était déjà plus une erreur géographique mais bien une catégorie politique vouant les nouveaux sujets amérindiens de Sa Majesté à un encadrement politique, religieux et fiscal, ne porte-t-elle pas bien davantage l'annonce d'une entreprise coloniale qui allait structurer un nouveau Monde? 1519, année du lancement depuis Cuba de l'expédition d'Hernán Cortés vers le Mexique, laquelle inaugurerait cette

* Jean-Noël Sanchez, Université de Strasbourg, membre du CHER UR 4376 et membre affilié de DynamE, UMR 7367. 
frénétique conquête continentale qui ferait tomber deux empires et soumettrait en faisant périr la plupart dans l'opération - des dizaines de millions d'hommes, n'est-elle pas plus éloquente quant à cet après lourd d'un avant anéanti ?

Cette année 1519, qui vit aussi Charles Premier d'Espagne être consacré cinquième du Saint Empire Romain Germanique, fut encore celle du départ de Sanlucar de Barrameda du renégat portugais Fernand de Magellan qui, en 1521, parviendrait enfin à rallier l'Extrême-Orient par la route occidentale que Colomb avait ouverte à ses illusions jamais détrompées. Mais si, en infraction manifeste du partage du monde réalisé à Tordesillas en 1494, Magellan atteignit bien pour l'Espagne l'archipel qui deviendrait les Philippines, ce ne fut qu'en 1565 que ce bout d'Asie insulaire devint effectivement espagnol. Ainsi, à défaut d'être acte, ou plutôt, parce que la concrétisation de l'acte qu'elle constitue serait différée de près d'un demi-siècle, cette découverte des Philippines, qui représente en somme l'aboutissement du projet initial de Colomb, est une date qui ne fait pas date, un non-événement au sens fort du terme.

Les Philippines seraient, donc, à partir de 1565, et avec elles un empire sur lequel, proverbialement, le soleil ne se couchait jamais. À l'instar de son homologue lusitain, le roi de Castille étendit son autorité Les quatre parties du monde (Gruzinski 2004), et chacun, au sein de l'hémisphère longitudinal qui lui avait échu, traça dans l'écume des immensités océaniques la moitié d'une couronne qui, en 1580, viendrait ceindre le royal chef d'un unique roi Philippe. Pourtant, si, du point de vue spatial, la Monarchie hispanique puis Ibérique semblait déjà inaugurer l'avènement de ce qu'on appelle aujourd'hui globalisation, il n'en va pas de même d'un point de vue temporel. Et là encore, ce furent ces Philippines reliées à leur métropole madrilène via la traversée de deux océans et d'un continent, qui constituèrent le point d'achoppement, l'extrême d'une ligne chronométrique trop tendue pour ne point rompre: la Monarchie Universelle se heurtait à l'impossibilité temporelle que représentait l'appréhension de cet archipel au bout de l'empire, de ces îles au bout du temps.

La colonie espagnole des Philippines et le rapport qu'elle entretint avec le temps durant son premier siècle, voici donc le propos des quelques pages qui vont suivre.

Elles seront subdivisées de la façon suivante. Dans une première partie, on mettra en évidence dans quelle mesure la mise en place de la colonisation des Philippines, qu'on la considère sous l'angle de la conquête séculière ou religieuse, se situe à un carrefour de temporalités hétérogènes. Dans un second temps, on tâchera mesurer l'impact de la durée sur la circulation des informations et sur la vie des hommes. Enfin, dans une dernière séquence, on s'attachera à penser le poids qu'eurent durant cette période les circonstances, les occasions manquées et le temps perdu. 


\section{L'espace du déjà : les Îles du Ponant, à la croisée de temporalités multiples}

\section{Le temps d'une conquête qui n'en est plus une}

1521, 1565, quarante-quatre années pour que les potentielles Philippines espagnoles devinssent réalité. Un demi-siècle, justement en un siècle qui marqua le début de la prodigieuse accélération du temps de l'Occident, un temps que l'Occident allait imposer au monde. Or, durant cette longue attente, l'Amérique surgit dans toute son ampleur, fut confrontée, vaincue, largement détruite, et sur ses cendres encore fumantes fut édifié un Monde Nouveau. Un appareil colonial fut mis en place de façon graduelle, à coup d'expériences, de retours en arrières, de formules enfin établies. En effet, étant donnée l'immensité et la diversité des espaces conquis, le processus de la conquête s'inscrivit dans une temporalité différentielle. Ainsi, lorsque commençait la conquête du Pérou, Mexico possédait déjà une Audience et Saint-Domingue une université.

La conquête à proprement parler correspond fondamentalement à la période comprise entre l'arrivée de Cortés au Mexique et la veille des premières tentatives d'exploration des solitudes amazoniennes, soit approximativement de 1520 à 1540. Dès avant ces vingt années de furie belliqueuse, Bartolomé de Las Casas dénonça les horreurs perpétrées par les chrétiens et, en leur milieu, Francisco de Vitoria, avec sa Relectio de Indis (Vitoria 1532) contribua résolument à l'approfondissement d'un débat sur la légitimité de la conquête qui prendrait fin avec la clôture de la fameuse controverse de Valladolid, en 1551. Dès lors, le terme de conquête perdit de sa superbe et devint disgracieux, comme en attestent déjà l'Instrucción sobre poblaciones y nuevos descubrimientos adressée en 1556 au marquis de Cañete, vice-roi du Pérou, et surtout les Ordenanzas de descubrimientos, nueva población y pacificación de las Indias promulguées par Philippe II en 1573 (Del Vas Mingo 1985). Désormais, la correction politique imposait de préférer les termes de "peuplement» ou de "pacification".

C'est à l'aube de ces temps nouveaux que se déploya donc l'occupation espagnole des Philippines. L'époque de la conquête était bel et bien révolue et ce ne furent plus des mercenaires qui, comme en Amérique, se lancèrent à l'assaut des Philippines, mais d'honorables officiers du roi, dirigés par Miguel López de Legazpi, qui avait derrière lui une carrière d'administrateur en Nouvelle-Espagne, un mariage avec la sœur de l'évêque de Tlaxcala, neuf enfants et soixante-trois années de vie. Signe de ce que l'époque n'était plus à la précipitation, il dut attendre jusqu'en 1568 pour recevoir son titre d'Adelantado, qui lui permit effectivement de prendre en charge la pacification de l'archipel. Cette conquête $[s i c]$ s'appuyait sur près d'un demi-siècle d'expérience sur le continent américain et, tout particulièrement, sur ce qui avait déjà été accompli au Mexique. C’est ainsi que Legazpi serait épaulé par les capitaines Juan et Felipe de Salcedo, ses petits-fils nés à Mexico. Désormais, les conquérants n'étaient plus des Espagnols mais des créoles nés aux Indes. 
Ils allèrent droit au but, et furent aidés en cela par une confluence structurelle des cultures amérindiennes et insulindiennes. En effet, l'institution politique centrale des ethnies de souche malaise qui constituent la majorité des populations de Luzon et des îles Visayas est le Barangay, qui tire son nom des embarcations qui ont permis aux ancêtres d'atteindre les Philippines. Elle établissait alors une division de la société en trois classes sociales, nobles, hommes libres et esclaves, qui ménageait à ceux qui en occupaient le sommet, les datu ${ }^{1}$, un solide pouvoir sur leurs subordonnés. En cela, la place du datu au sein du barangay était tout à fait comparable à celle du cacique sur laquelle l'entreprise coloniale espagnole avait pu asseoir en Amérique le nouveau pouvoir en s'appuyant sur le relais des autorités indigènes locales. Manille fut conquise dès 1571 et obtint à peine trois ans plus tard le titre de Noble y Siempre Leal Ciudad. En quinze ans, pas moins de 241 encomiendas (mise sous tutelle d'un groupe d'indigènes) furent distribuées aux conquérants (Abásolo, 2000). Aux environs de 1590, les territoires effectivement contrôlés à Luzon, l'île de Manille et dans les îles Visayas, au centre de l'archipel atteignait déjà les des deux tiers de la superficie de la région ${ }^{2}$.

En outre, cette colonisation des Philippines fut l'occasion d'un singulier processus multidirectionnel de translation et re-sémantisation des rapports que les Espagnols avaient historiquement entretenu avec des Autres.

Les serviteurs de Philippe II s'empressèrent en effet de désigner les indigènes de l'archipel en faisant usage du terme d'Indio ${ }^{3}$, un exonyme, fruit de la célèbre confusion colombine, qui avait déjà été généralisé à tous les groupes natifs des Indes Occidentales. La distance et l'ignorance aidant, il n'est ainsi pas rare de rencontrer au détour d'un document produit par Madrid la mention de quelque Indio chino ou Indio japón, ce qui, au-delà de l'incongruité géographique finalement moins abyssale que dans le cas d'un mal-nommé Indio du Paraguay, démontre à quel point l'expérience américaine du passé récent avait puissamment conditionné le rapport que les Espagnols devaient entretenir avec le monde extra-européen. Il est d'ailleurs significatif de constater que ce processus d'assimilation sémantique, de translation d'un temps et d'une réalité vers une autre, allait aussi concerner des ethnies concrètes. C'est ainsi que les Zambales, ces frustes indigènes insoumis vivant dans les collines et qui fondaient périodiquement sur les villages des plaines où les indigènes christianisés cultivaient le riz nécessaire à la subsistance de la colonie, furent promptement assimilés aux Chichimèques, leur équivalent mexicain, comme en témoigne explicitement la relation de Miguel de Loarca de 1582 (Archivo General de Indias, Patronato, 23, 9).

1 Le terme datu signifie littéralement «riche», comme dans le titre malais orang kaya.

2 Il convient néanmoins de noter que, dans la région centrale de Luzon de la Cordillera, ce n'est qu'à la fin du XIX ${ }^{e}$ siècle que les Espagnols parviennent à imposer un tant soit peu leur autorité sur les ethnies Bontoc et Ifugao.

3 Les Indiens de l'Inde sont quant à eux appelés d'ordinaire Malabares. 
Mais il y a plus frappant encore. Les Chinois, ou Sangley comme les Espagnols les nommèrent, pour la plupart originaires du Fujian, d'où ils furent attirés par l'argent du Mexique que les nouveaux venus semblaient receler en abondance, affluèrent rapidement vers Manille. Vivant pour la plupart au sein d'un quartier séparé de la ville espagnole, le Parian, à l'instar des ghettos des grandes villes péninsulaires du Moyen-Âge, ils furent rapidement l'objet de préjugés concernant leur supposée avidité, mais aussi d'une constante méfiance vis-à-vis des manigances que la communauté soudée qu'ils constituaient était susceptible d'ourdir contre les chrétiens. En 1603, 1639, 1662, dates auxquelles le Parian s'embrasa, le schéma fut toujours le même. On s'inquiéta subitement du nombre prodigieux de Chinois qui faisaient face à une bien modeste communauté espagnole, des rumeurs de complot suscitèrent bien vite le harcèlement des colons; s'ensuivit un soulèvement, puis un massacre auquel participèrent alégrement les autres communautés asiatiques, suivi d'une expulsion, puis d'un retour à la situation antérieure face à l'impossibilité de vivre sans ces indispensables protagonistes de la vie économique manillaise.

On voit clairement la similitude de ce rejet avec l'antisémitisme dont l'Espagne avait été et continuait plus que jamais à cette époque à être animée. On peut ainsi mentionner une cédule royale de 1643 répondant à la lettre du gouverneur Corcuera (1535-1644) dans laquelle celui-ci proposait d'obliger les Sangley à résider derrière des murs, "comme pour les juifs" de Rome et d'Alexandrie (A.G.I., Fil., 330, 4, fo 155v-156r) ${ }^{4}$.

Mais c'est assurément le cas des indigènes musulmans du sud de l'archipel, lesquels opposeraient une résistance aussi farouche que durable aux colonisateurs hispaniques ${ }^{5}$, qui constitue ici le cas le plus manifeste, systématique et pérenne de duplication catégorielle. En effet, quoique bien éloignées de la Berbérie, ces autochtones, par ailleurs très proches culturellement des Indios que les Espagnols s'attachaient à convertir en de bons chrétiens, furent immédiatement qualifiés de Moros, comme jadis les musulmans qui occupèrent la Péninsule.

L'expérience péninsulaire de la reconquête avait déjà profondément conditionné la première occupation de l'Amérique (Bernand \& Gruzinski 1991). Des institutions telles que l'encomienda et le repartimiento, qui constituèrent tout à la fois le ciment du système d'exploitation de la main d'œuvre indigène et le versant matériel du statut légal de l'Indio, virent ainsi le jour au XIII ${ }^{\mathrm{e}}$ siècle, en vue de prendre en charge les populations musulmanes de la vallée du Guadalquivir. On peut à cet égard encore mentionner la première Carta de

4 Voir aussi Gil 2011. Deux siècles plus tard, dans le rapport intitulé Presupuestos de gastos e ingresos de las islas de Cuba, Puerto-Rico y Filipinas para el año 1839 (1840: 61) Madrid, Imprenta Nacional, 1840, p. 61, on inviterait à la prudence vis-à-vis de la communauté chinoise, qui forme "una especie de liga, semejante a la de los judíos, con quienes tienen mucha analogía en sus principios sociales y mercantiles».

5 Pour s'en convaincre, on peut visiter la salle consacrée à leurs artefacts guerriers au Museo Naval de Madrid. 
relación d'Hernan Cortès du 6 juillet 1519, dans laquelle le conquérant désignait les temples mésoaméricains sous le nom de «mezquitas», ou encore la rapide mutation sous les cieux américains de Santiago «matomoros» en Santiago «mataindios» (Domínguez García 2006). Plus généralement, l’idée prophétique en vertu de laquelle, en leur permettant de découvrir de nouvelles terres, Dieu avait voulu que les Castillans pussent continuer toujours à lutter contre les infidèles, constituait pour ainsi dire un fait établi dans l'Espagne de la première moitié du Xvi ${ }^{\mathrm{e}}$ siècle.

Cette articulation entre reconquête et conquête se fit outrancière aux Philippines, certains contemporains allant jusqu'à établir un rapport de cause à effet factuel entre les deux phases historiques. C'est ainsi qu'en 1584, l'étonnant licenciado Melchor Dávalos exposa ses réflexions «au sujet de ce qu'on fera des mahométans de ces terres et mers». Appuyant son argumentation sur d'abondantes références scholastiques, celui-ci proposait de lancer une offensive massive contre les places musulmanes de la région et, afin de convaincre les fonctionnaires du Conseil des Indes, arguait que les mahométans asiatiques s'étaient confédérés avec des «turcs, des mamelouks, des maures de Tunis et des maures qui avaient été expulsés de grenade à l'Époque des Cois Catholiques ${ }^{6}$. Et si l'expédition que Dávalos proposait d'organiser à ses frais avec ses fils ne fut pas validée, en 1595, le capitaine Esteban Rodríguez de Figueroa put quant à lui lancer une authentique conquête de Mindanao, la grande île du sud de l'archipel, sur la base de capitulations typiques de l'époque alors révolu où Couronne et condottières établissaient des contrats fixant les modalités des futures entreprises d'expansion.

C'est donc bien une nouvelle réplique paradoxale du passé espagnol qui allait se jouer ici. Durant plusieurs siècles, les indigènes christianisés des villages côtiers des îles Visayas allaient vivre dans l'angoisse des razzias musulmanes portées par les courants venant du Sud. Les religieux espagnols en mission dans la région, toujours prêts à sonner le tocsin pour que leurs ouailles se réfugiassent dans des églises aux allures de forteresses et dont les clochers faisaient office de tours de garde (bantayan moro), allaient importer dans l'archipel des Comedias de Moros y Cristianos, ces pièces de théâtre issues de la tradition populaire hispanique qui relatent avec candeur de fort vieilles histoires de princesses enlevées, de maures perfides et de batailles héroïques. Une fois acclimatées à la chaleur moite des Philippines sous le nom chantant de moro-moro, sans pour autant que cela impliquât un quelconque changement de cadre de l'intrigue et de protagonistes, celles-ci allaient devenir l'«authentique» expression artistique

6 Notre traduction, comme les citations traduites qui suivront. Lewis Hanke (1943) publia deux écrits de Dávalos. Le texte qui nous semble pour notre part le plus représentatif de la perspective de l'oidor est sa lettre du 3 juillet 1584, A.G.I., Filipinas, 18a, 2, 9. Sur la perception espagnole des musulmans asiatiques aux Xvi ${ }^{\mathrm{e}}$ et $\mathrm{XVII}^{\mathrm{e}}$ siècle, voir Jean-Noël Sanchez (2017). 
locale d'un conflit qui s'était joué avec d'autres protagonistes, autour d'autres enjeux, à une autre époque et sous d'autres cieux ${ }^{7}$.

\section{Le temps des moines}

L'entreprise de colonisation de l'archipel se construisit ainsi très largement depuis l'Amérique, qui lui constitua un passé sur lequel s'appuyer avant que son temps ne vînt. La chose est d'autant plus flagrante s'agissant du processus d'évangélisation des Philippines, cette autre conquête qui, quant à elle, put assumer son nom, et qui allait assurément constituer le grand orgueil et aussi, d'un certain point de vue, la grande réussite de l'Espagne aux Philippines.

En 1565, deux ans après la clôture du Concile de Trente, voulu et inauguré par Charles Quint et finalisé sous l'égide de son fils Philippe II, le débat sur la légitimité de la conquête avait donc déjà eu lieu. À l'issue de celui-ci, c'était bien la mission évangélisatrice de l'Espagne qui s'imposait comme la seule vraie justification de la prise de possession espagnole sur les terres nouvelles.

À cette première temporalité fondatrice s'ajoute une seconde, européenne celle-là: le début de la Guerre de Quatre-Vingt ans contre les rebelles néerlandais protestants, trois ans à peine après l'installation aux Philippines. Dans le cadre de ce conflit, les Bataves réformés allaient lancer une authentique guerre idéologique, en s'appuyant notamment sur la Brevísima relación de la destrucción de las Indias de Bartolomé de Las Casas (1551), court écrit dans lequel le dominicain dénonçait avec des accents apocalyptiques les horreurs perpétrés par les Espagnols aux Amériques. À partir de 1578, ce texte, accompagné pour l'occasion des terrifiantes gravures de Théodore de Bry, allait connaître une véritable fortune éditoriale dans le nord de l'Europe ${ }^{8}$. Dès lors, le prince catholique devrait démontrer qu'il agissait dans une perspective nonmachiavélienne ${ }^{9}$, c'est-à-dire non pas dans son seul intérêt, mais pour le bien de ses sujets exotiques. Or, quel bien plus précieux que le salut de l'âme et quel espace pour mettre mieux en exergue cette juste tutelle exercée par l'Espagne que les îles baptisées du nom de son souverain?

L'historien jésuite Francisco Colín ouvrit son Labor Evangélica en affirmant: «El señor Felipe Segundo...decía que por una sola Ermita, en que en Filipinas se conservase el Santo Nombre de Dios gastaría la renta toda de sus

7 Sur le phénomène européen, on peut consulter Albert-Llorca \& González Alcantud (2003). Pour l'Amérique, mentionnons entre autres travaux l'étude classique de Ricard (1932), et Cáceres Valderrama (2005). Sur les Philippines, voir Retana (1909), et bien plus récemment Isaac Donoso (2010).

8 En 1578, le livre parait pour la première fois en néerlandais et l'année suivante en français. Entre 1578 et 1700, ce ne sont pas moins de 62 rééditions qui allaient voir le jour.

9 Un texte central dans la mise en place de cette argumentation est le traité du jésuite Pedro de Ribadeneyra (1595). 
Reinos $»^{10}$ (Colín 1663). Certainement apocryphe, cette citation n'en rend pas moins compte d'une réalité. Les Philippines eurent le piètre privilège d'être une des rares colonies déficitaires de la monarchie. Cette déplaisante réalité se rajoutant aux contraintes logistiques attenantes à la seule colonie espagnole d'Asie, on conseilla souvent aux rois Philippe d'abandonner les Philippines, ce qui leur donnait immanquablement l'occasion de clamer la primauté des impératifs spirituels sur les considérations matérielles, comme en témoigna en 1621 le secrétaire du bureau de la marine Martínez de Aróstegui:

Chaque année on dépense aux Philippines plus de trois-cent mille ducats pour mener la guerre contre les maures et les hérétiques septentrionaux; et bien qu'on a présenté à Sa Majesté des propositions pour abandonner ces îles, seulement afin que ne soit pas abandonnée la chrétienté nombreuse qu'elles abritent et la moisson récoltée pour la Foi par l'intermédiaire des ouvriers [de Dieu] qu'il a envoyé, il n'a pas voulu le faire, mais a plutôt envoyé des secours au prix d'une grande dépense. (Real Academia de la Historia 1889)

Pour la Monarchie Catholique, le temps des Philippines était donc sans ambigüité: il devait être le temps de Dieu, celui qui justifierait un passé contestable par les probes intentions faites réalité dans un présent sacrificiel continuellement reconduit.

Le premier synode de Manille fut inauguré en 1581. Son instigateur, le dominicain Domingo de Salazar, diplômé en droit civil et canonique à l'université de Salamanque, fort d'une expérience missionnaire de 23 ans au Mexique et en Floride, se confessait volontiers fervent admirateur de son illustre coreligionnaire. Les religieux qui participèrent à l'événement ne tâtonnèrent guère. Dès le préambule, le nouveau décor fut fermement planté. Au Mexique, au Pérou, la prise de possession espagnole était un fait accompli. Aux Philippines en revanche, tout était encore possible. La question de la légitimité de la conquête ne s'y posait donc pas après-coup mais bien in media res. Si elle ne pouvait être justifiée, les Espagnols devaient immédiatement rendre ce dont ils s'étaient indument emparés et réparer autant que faire se pût les dommages infligés aux populations sous peine d'être en état de péché mortel. Se fondant sur les arguments développés par Vitoria, dominicain lui aussi, les religieux en vinrent logiquement à poser la présence politique de l'Espagne aux Philippines comme étant assujettie à l'objectif d'évangélisation, in ordine ad finem spiritualem (Gutiérrez 1992).

Les îles du Ponant allaient donc être, par excellence, terre de mission, une vocation qui porterait ses fruits puisque, aujourd'hui, le pays constitue la troisième communauté catholique mondiale.

Les débuts furent pourtant difficiles, du fait d'une troisième synchronie: la découverte de la Chine et les espoirs de conversions multitudinaires au sein d'une civilisation admirable que celle-ci suscita. Dans un premier temps et

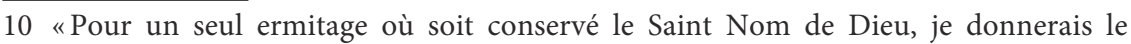
bénéfice de tous mes Royaumes». 
jusqu'au début des années 1590 ce fut donc l'Empire du Milieu qui cristallisa les rêves millénaristes des religieux. Nonobstant, le nombre de missionnaires présents dans l'archipel grossit rapidement. En 1576, il y avait 13 missionnaires aux Philippines. Dix ans plus tard, ils étaient 94, et 267 en 1594. Le grand tournant de l'évangélisation des Philippines se situa précisément au milieu de la décennie, en 1595, année de création de l'archidiocèse, des trois diocèses provinciaux de Nueva Segovia, Nueva Cáceres et de Cebu, et de la répartition du champ missionnaire philippin entre les divers ordres en vertu d'une logique ethnolinguistique. La «christianisation rapprochée» qui serait développée aux Philippines pouvait désormais commencer. Car si la spectaculaire vitesse du rythme des conversions indigènes au christianisme, 280000 en 1594, 322000 en 1612, 500000 en 1626 (Phelan 1959:53-71), semble de prime abord incompatible avec une évangélisation sourcilleuse qui se fût donnée le temps, on peut pourtant assurer que les religieux présents dans l'espace philippin parvinrent rapidement à donner aux grands moments de la vie catholique une forte prégnance dans la vie des indigènes philippins, à tel point qu'on peut certainement accréditer les propos de l'historien jésuite Pedro Chirino qui affirma au début du XvII ${ }^{\mathrm{e}}$ siècle que la formation religieuse des indigènes philippins était meilleure que celle dont de nombreuses populations européennes pouvaient disposer à la même époque (Chirino 1604).

À la longue, pourtant, ce qui avait fait des Philippines un espace à la pointe de son temps convertirait l'archipel en une contrée marquée par un profond archaïsme. Ainsi, dès la seconde moitié du XVII siècle, l'encomienda majoritaire dans l'archipel était entre les mains d'ordres religieux qui parvinrent dès lors à se constituer de vastes domaines agraires sur Luzon. En position de force dans les provinces où les Espagnols laïcs étaient peu nombreux, ils imposeraient leur loi sur les vies autochtones. Dans une certaine mesure, les Philippines allaient être prises au piège de leur exemplarité. Dès lors, en dépit des tentatives de plusieurs gouverneurs de déconfessionnaliser la présence espagnole dans le pays, le constat qu'à la fin du XIX ${ }^{\mathrm{e}}$ siècle établiraient les ilustrados, de jeunes intellectuels issus de la classe indigène aisée qui s'attacheraient à penser le devenir de leur patrie, serait sans appel: les Philippines étaient bel et bien devenues une «monacocratie» (Del Pilar 1889).

\section{L'espace de l'encore: La mesure du temps d'un extra-lointain colonial}

\section{La temporalité de l'écriture}

Des papiers, telle est la matière première de l'historien.

Des papiers, à l'odeur des vieilles choses, souvent rigides au toucher, du fait du sel marin qui en a pénétré les pores, fragiles, de sorte que, parfois, il faut ganter ses mains pour les manipuler. Des papiers en prodigieuse quantité, à l'Archivo General de Indias de Séville, dans la belle bâtisse de la Casa de Contratación, à deux pas de la Giralda, qui abrite la documentation issue de tout l'espace colonial jadis dominé par l'Espagne. À l'Archivo General de la 
Nación, à Mexico, en service depuis 1746, et situé aujourd'hui dans le palais de Lecumberri, qui fut jusqu'en 1976 une prison ${ }^{11}$, lequel, certes, ne recèle «que» les fonds de l'ancienne vice-royauté de Nouvelle-Espagne, mais peut néanmoins se prévaloir des précieux 1773 volumes d'enquêtes de l'Inquisition mexicaine et de ses satellites. Des papiers, on en trouve en bien d'autres endroits encore. La tatillonne administration de la monarchie hispanique du siècle d'or est en effet une énorme productrice de papiers (Gaudin 2013).

Ces papiers ont bien sûr un contenu, forcément divers, mais d'ordinaire rédigé au format imposé par des conventions d'écritures, des formules préétablies et un vocabulaire convenu, toutes choses qui, il faut l'avouer, facilitent la tâche de celui qui doit déchiffrer les volutes alambiquées et les sibyllines abréviations de quelque obscur fonctionnaire de Sa Majesté.

Ces papiers, enfin, ont une temporalité1 ${ }^{12}$. C'est une date sur une lettre, qui témoigne du moment où tel gouverneur - ou son secrétaire - prit la plume pour écrire ce dont il fallait qu'il informât ou rendît raison, où tels administrateurs du Conseil des Indes décidèrent d'informer ou de demander des informations à ce gouverneur, pour évoquer la configuration la plus fréquente. En réalité, ces courriers ont sans doute été pensés à un autre moment, puisque plusieurs d'entre eux portent souvent la même date, laquelle précède de peu le moment où le courrier entama le long itinéraire qui le mena à son destinataire ${ }^{13}$.

Au bout d'un certain temps, à force de fréquenter ces vieux papiers, on prend subitement conscience de ce qui est pourtant une évidence: cette date se tient dans un équilibre instable entre deux autres dates.

La première de ces bornes temporelles, c'est la date du document qui, aux deux sens du terme, correspond avec celui qu'on consulte, la missive du fonctionnaire à laquelle répondit la Couronne, l'ordre royal auquel réagit le fonctionnaire. Dans la majorité des cas, elle est mentionnée au début du document, afin que l'interlocuteur pût savoir aussitôt de quoi il en retournait. Les archivistes, dans un espace-temps qui vient se superposer à celui du document et de l'investigateur, ont souvent eu la bonne idée de classer ensemble les deux écrits. Si ce n'est pas le cas, il faut alors chercher, quelques fois sans trouver.

La seconde de ces bornes, c'est la date de réception de l'écrit en question, laquelle est généralement inconnue, quoique vraisemblablement peu éloignée

11 C'est là aussi la première vocation du château qui abrite l'Archivo General de Simancas.

12 Sur la question de l'espace-temps dans l'administration coloniale, on lira avec grand profit Rivero Rodríguez \& Gaudin 2020.

13 La Lilly Library de l'Université de l'Indiana à Bloomington possède le libro de gobierno de Sebastián Hurtado de Corcuera, rédigé par son secrétaire de juillet 1641 à janvier 1642, Philippines MSS, LMC 1832. Mise en reflet avec les lettres adressées par le même gouverneur au Conseil des Indes, l'étude future de ce document, unique en son genre, pour ce qui est des Philippines tout au moins, permettra peut-être d'éclaircir en quelque manière le processus de rédaction des documents destinés à informer Madrid des événements de la colonie. 
de la date de la réponse. Encore faut-il qu'on ait considéré qu'il convenait de répondre au plus vite. Ce qui n'était pas toujours le cas. Ainsi, en 1607, le procurador general des Philippines Hernando de los Ríos Coronel écrivit à Madrid au sujet de l'opportunité de réduire en esclavage les Indios mahometanos capturés dans le cadre d'une guerre juste (A.G.I., Fil., 27, 63). Cependant, laisseraller propre à l'administration du duc de Lerma oblige, ce ne fut qu'en 1620, au moment où Baltasar de Zúñiga et son neveu Gaspar de Guzmán, le futur comteduc d'Olivares, prirent les rênes de la monarchie avec le projet d'en restaurer l'efficacité et le prestige, que la proposition fit l'objet d'une cédule royale qui la validait (A.G.I., Fil., 340, 3, fo 266r-267r).

Ce prodigieux délai de treize années est bien sûr exceptionnel. Mais le flux des allers et retours de correspondance n'en était pas moins assujetti à une durée difficilement compressible: le temps du voyage.

Une seule flotte, constituée d'un à quatre galions, quittait annuellement Manille pour rejoindre Acapulco, d'ordinaire entre la mi-juin et la mi-juillet, du fait des contraintes climatiques imposées par la mousson et la saison des ouragans. La traversée du plus grand des océans de Manille à Acapulco durait en moyenne six mois. Parfois plus, et même trop. Ainsi, en 1657, un galion, probablement le San José premier du nom, fut aperçu à la dérive au large des côtes mexicaines de Huatulco. Parti des Philippines plus d'un an plus tôt, il n'y avait à son bord plus âme qui vécût. Une fois le port mexicain atteint, il fallait encore que les missives fissent le voyage terrestre jusqu'à la capitale de la viceroyauté. De là, un nouvel itinéraire terrestre jusqu'à Vera Cruz, d'où sortaient deux flottes par an pour Séville, ce qui imposait une nouvelle odyssée de quelques trois mois (Chaunu 1969: 285). Ne restait alors qu'à atteindre Madrid. Le voyage retour impliquait évidemment d'entreprendre le même itinéraire dans l'autre sens, avec d'autres conditions de navigation: près de 130 jours pour le voyage vers la Nouvelle-Espagne, mais seulement trois mois pour que la nao de Acapulco n'atteignît les Philippines, d'ordinaire au mois de mai ou juin, pour la plus grande joie de la petite communauté espagnole d'Asie pour laquelle apercevoir les voiles des galions au fond de la Baie de Manille constituait assurément l'événement le plus important de l'année (Schurz 1939: 251-283).

D’après Pierre Chaunu, ce n'était pas moins de cinq longues années qui étaient nécessaires afin d'accomplir le voyage entre l'Espagne et les Philippines dans un sens et dans l'autre (Chaunu 1969: 277). Il y a là certainement quelque surenchère et, en ce qui concerne la pérégrination de l'information, on peut sans doute ramener la moyenne d'un aller-retour de correspondance à un peu moins de trois ans, lorsqu'aucun incident ne venait troubler cette longue migration $\mathrm{du}$ papier. Il pouvait en effet se présenter des incidents de navigations, soit que le navire coulât corps et biens, soit que, par suite d'une avarie (arribada), il dût rebrousser chemin, ce qui résultait presque aussi néfaste en termes financiers et communicationnels. Ainsi, pour la première décennie du XVII ${ }^{\mathrm{e}}$ siècle, William Schurz (1939: 258), auteur du classique The Manila Galeon, ne dénombra pas moins de six naufrages. Cinquante ans plus tard, en moins de dix ans, 
trois naufrages $(1653,1654,1661)$ et deux voyages avortés à cause d'avaries $(1655,1660)$ furent à déplorer (Prieto Lucena 1984: 44-52). Dans ces cas-là, le voyage se trouvait fatalement repoussé d'un an. Les problèmes de transport ne furent d'ailleurs pas l'apanage de la route Pacifique et pouvaient tout aussi bien se présenter dans l'Atlantique, comme d'ailleurs en tout point de ce périple entre trois continents. Ainsi, le 15 décembre 1614, dans un contexte où la communication entre les Philippines était impérieuse, Madrid écrivit au gouverneur des Philippines pour lui demander des nouvelles des îles en l'absence de courrier en provenance de l'archipel postérieur à ses lettres de juillet 1612, du fait de l'absence de navires en provenance de la Nouvelle-Espagne en 1613 (A.G.I., Fil., 329, 2, fo 185r).

Or, la lenteur des communications était d'autant plus dommageable pour la monarchie hispanique que celle-ci constituait une énorme machine administrative extrêmement centralisée. Un fonctionnaire de Sa Majesté ne pouvait en aucun cas prendre une décision de nature organisationnelle sans en référer expressément à Madrid, moyennant l'étape de la vice-royauté mexicaine s'agissant de la colonie des Philippines.

En comparaison, l'Estado da Índia portugais, sur les bases d'une tradition législative beaucoup plus lâche, était indéniablement plus décentralisé: le viceroi y jouissait d'une large marge de manœuvre pour administrer l'Asie, tandis qu'une cité comme Macao, en quelque sorte la sœur jumelle de Manille, ne dépendrait d'aucun représentant officiel de Goa jusqu'en 1623, significativement par suite d'une intervention espagnole (Videira Pires 1987: 20-21). Quant à la Compagnie des Indes Orientales néerlandaise, la VOC, qui deviendrait à partir de 1602, date de sa création, et jusqu'au milieu du XVII ${ }^{\mathrm{e}}$ siècle, le pire cauchemar asiatique des Ibériques, les seuls comptes que son gouverneur général devait rendre à Amsterdam étaient justement des livres de comptes, tandis qu'il avait toute latitude pour établir des accords diplomatiques et même déclarer des guerres de son propre chef.

Pour leur part, les organes centraux de la monarchie catholique exigeaient expressément une intense production scripturale dans laquelle il est difficile de ne pas déceler un acte dont la valeur était largement performative. Si l'État pouvait obtenir d'abondants rapports produits par des sujets distants de lui de plusieurs milliers de lieues, c'est qu'il existait bel et bien en ces confins reculés, et que l'empire était donc une réalité, ne fût-ce que de papier. À l'autre bout du monde, lesdits individus de la périphérie pouvaient quant à eux à bon droit présumer que, dans la mesure où ils écrivaient copieusement au centre et que, ce faisant, ils entérinaient son modus exprimendi et sacrifiaient à ses rites, leur obéissance à son égard était implicitement validée, ce qui laissait toute marge de manœuvre pour faire par ailleurs en sorte que ce ne fût pas forcément le cas. C'est là le sens du fameux «se acata pero no se cumple», "on obéit mais on n'exécute pas », formule proverbialement utilisée pour décrire la particulière relation que les colons d'Amérique ont historiquement entretenu avec la Couronne. 
Depuis les Philippines, il y avait en outre particulièrement à demander ou à justifier, puisque, comme on l'a dit précédemment la colonie était lourdement déficitaire, de quelques deux-cent cinquante mille pesos annuels, soit près d'un douzième des revenus perçus par l'Espagne pour toute l'Amérique ${ }^{14}$, et avait donc crucialement besoin d'argent, mais aussi de soldats et de matériel militaire. Sa configuration singulière imposait de même plus d'explications, puisqu'on comprend aisément que les dispositions légales concernant les indiens des Indes Occidentales exigeaient quelque adaptation pour qu'elles fussent applicables au cas des Chinois de Manille ou des Moros du sud de l'archipel.

Incerta lex sed lex. En réalité, mises à part les Leyes de Burgos de 1512 et les Leyes Nuevas de 1542 qui concernaient les mesures de mises sous tutelle et de protection des indigènes, il n'y eut pas à proprement parler de corpus légal spécifique à l'espace colonial espagnol jusqu'à la fin $\mathrm{du} \mathrm{XVII}{ }^{\mathrm{e}}$ siècle et la rédaction de la Recopilación de las Leyes de los Reynos de Indias (Pinelo \& Solorzano 1681). Celle-ci synthétisa en effet les milliers de dispositions qui avaient été établies par le biais de cédules royales depuis le début de la mise en place de l'autorité politique espagnole aux Amériques. C'est ainsi que, par exemple, dans le Livre Six, Titre Premier, Des Indiens, sous l'item «Ley XXXI, «Qu'on ne puisse vendre des armes aux Indiens, et qu'ils ne peuvent pas en posséder», on peut lire: «D. Fernando V et Doña Isabel à Grenade le 17 septembre 1501. L'Empereur D. Carlos le 16 février 1536 et le Prince Gouvernant à Madrid le 17 décembre 1551. D. Felipe II le 25 janvier 1563 et le 10 décembre 1566, et le 18 février 1567 et le $1^{\text {er }}$ mars 1570 ».

Cette loi résulte donc d'une synthèse de différentes cédules royales se situant dans des réalités spatio-temporelles diverses, puisqu'il s'agit de dispositions ayant répondu à un problème précis qui s'est présenté à un moment donné en quelque province des Indes de Sa Majesté. Conséquemment, avant l'établissement de la compilation, les fonctionnaires espagnols, qu'ils fussent établis dans la métropole ou dans les confins du globe, devaient penser l'ordre colonial par le biais de ces bribes d'espace-temps singuliers qui constituaient des règles générales en puissance. On comprendra aisément que, la distance, les délais des échanges et les changements de personnel administratif aidant, les contemporains s'y perdaient aisément.

En 1615, le gouverneur Juan de Silva, en conflit avec les fonctionnaires de l'audiencia, le tribunal faisant office de gouvernement local d'une province de l'empire, contre lesquels il allait d'ailleurs réunir un imposant dossier de plus de 2000 pages (A.G.I., Fil., 63, 1), écrivit ainsi au Conseil des Indes pour lui faire part de ce qu'on lui avait rapporté que les oidores (les membres de l'audiencia) seraient en possession d'une cédule leur permettant de mobiliser la population espagnole de l'archipel sans devoir en référer au gouverneur et potentiellement contre lui, ce pour quoi il demandait l'émission d'une cédule invalidant l'hypothétique premier document. Madrid ne répondit pas par une cédule

14 Calculs effectués à partir des chiffres proposés par Morineau 1978: 151-152. 
officielle, mais par une lettre secrète aux membres de l'audiencia dans laquelle il leur fut demandé de ne faire en aucun cas usage de cette cédule, au cas où celleci existerait vraiment, puisque, perdus dans des masses de documents, parfois contradictoires entre eux, que leurs prédécesseurs avaient établis en réponse à des missives lointaines dont eux n'avaient pas connaissance, les fonctionnaires du Conseil n'en avait tout simplement aucune idée (Schäfer, 2003: II, 149, note 473; A.G.I., Fil., 20, 9, 56)!

Deux ans et demi ou trois ans, la durée de l'aller-retour informatif était, dans tous les cas, trop longue ou, pour le moins, suffisamment longue pour que les informations communiquées fussent devenues totalement caduques. Dès lors, si au XVI ${ }^{\mathrm{e}}$ siècle, a fortiori après 1580, lorsque Philippe II devint roi du Portugal et de ses territoires d'outre-mer, l'Espagne constituait un empire mondialisé, celuici ne saurait encore, loin s'en faut, être considéré comme un espace ibérique globalisé, du fait de cette limite infranchissable qu'imposerait encore pour longtemps encore la durée des communications.

Cet état de fait est d'autant plus patent si on considère une autre durée, celle durant laquelle ses hommes étaient chargés de produire ces documents. Pour continuer sur le cas des gouverneurs, la durée moyenne de leur mandat entre 1565 et 1663 se situe à quatre années ${ }^{15}$. Cela signifie que, dans la majorité des cas, les instructions qu'ils recevaient étaient des réponses aux rapports ou demandes de leurs prédécesseurs tandis que, passée leur première année de mandat, les chances étaient maigres qu'ils eussent l'opportunité d'agir en conséquence des réponses royales à leurs propres demandes. Il y a là assurément une bien étrange configuration dont il n'est pas aisé de déterminer le poids sur les consciences et la portée.

De même, comment comprendre dans un tel contexte la notion d'urgence? Ainsi, dans une lettre au roi du 4 août 1628, le gouverneur Juan Niño de Tavora (1626-1632), après avoir souligné que la colonie ne survivait que grâce à des tours de magie («vive por arte de encantamiento»), et que les secours reçus de Nouvelle-Espagne ne suffisaient pas, concluait son propos en évoquant:

ce gouvernement pour lequel je me sens dans l'obligation de supplier Votre Majesté avec l'humilité nécessaire de bien vouloir me retirer au cas où il n'y ait pas moyen de le secourir comme je le demande car je suis très sûr de la hâte avec laquelle il court à sa Ruine et il n'est pas juste que, entre les mains d'une personne avec les obligations et intentions qui sont les miennes, une chose de si grande importance pour l'Église Romaine et la couronne de Votre Majesté se perde. (A.G.I., Fil., 8, 1, 5)

15 Ce résultat, impliquant les mandats de vingt gouverneurs, titulaires ou intérims, depuis Miguel López de Legazpi (1565-1572) jusqu’à Sabiniano Manrique de Lara (1553-1663). Notons que cette moyenne se trouve largement gonflée par les trois derniers mandats, respectivement 8,9 et 10 ans et que nous avons pris à chaque fois comme référence de fin de mandat non pas la nomination du successeur mais le départ effectif. 
«La hâte»... Dans quelle mesure cet homme d'un autre temps, lorsqu'il écrivait ces mots, pouvait-il ignorait que Cronos allait inexorablement dévorer, non pas la matière - du moins est-elle parvenue jusquà nous - mais le sens même de ce qu'il était en train d'écrire?

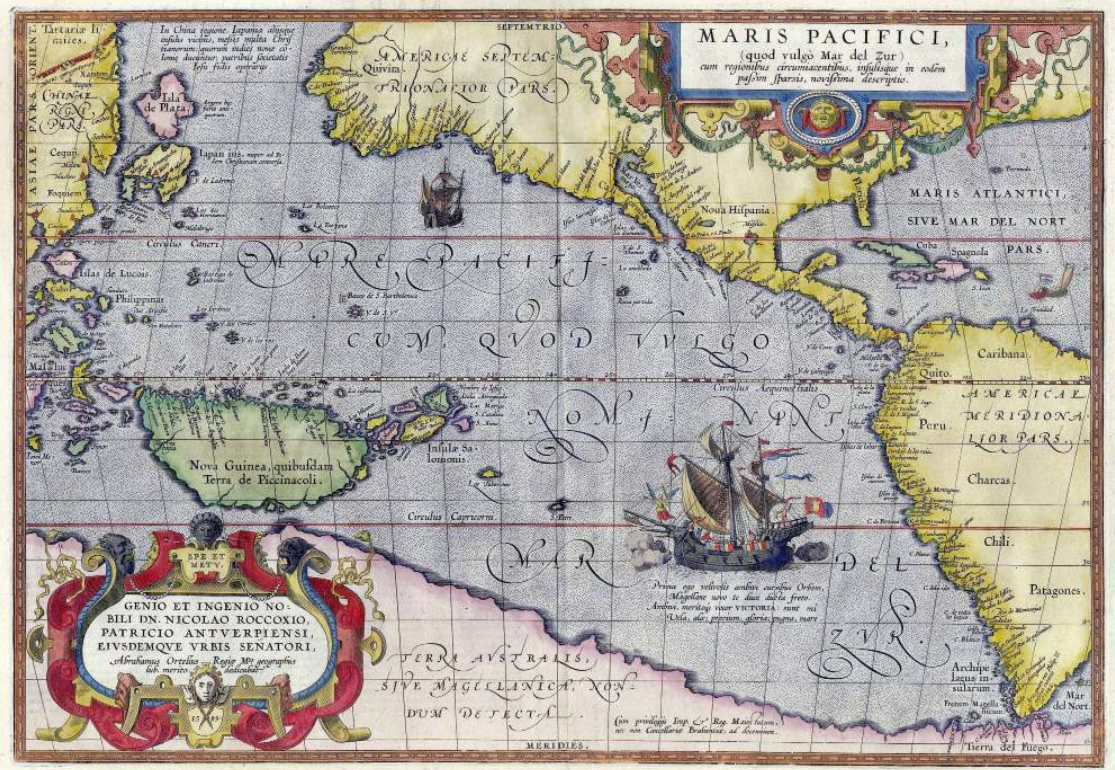

Abraham Ortelius, Maris Pacifi, 1585. Carte reproduite avec l'aimable autorisation de Helmink Antique Maps.

\section{Des vies d'hommes au long cours}

Les navires transportaient des papiers, des marchandises, mais aussi et surtout des vies d'hommes.

À l'époque, l'espérance de vie en Europe ne dépassait guère les quarante ans, et les occasions de mourir pour des individus traversant deux océans pour rejoindre une contrée à bien des égards hostile étaient nombreuses. Pierre Chaunu estimait que les chances de réaliser l'aller-retour Séville-Manille ne dépassait pas les 30\% (Chaunu 1969: 278). Là encore, on peut suspecter quelque exagération que seuls des calculs précis, comme l'auteur le reconnaissait luimême, sauraient ou non corroborer. Il n'en demeure pas moins que, sur les vingt gouverneurs des Philippines de 1565 à 1663, sept moururent avant d'avoir pu quitter l'archipel. Quant au risque de perdre la vie en mer, on aura compris qu'il est considérable. En 1600, en route pour Acapulco, le Santo Tomás essuya une série de tempêtes avant d'atteindre en piteux état les îles Mariannes: 50 survivants sur 260 passagers, soit la disparition de plus d'un dixième de la population espagnole de l'archipel à cette époque (Phelan 1959: 100; Corpuz 1997: 46). Le San Nicolás sombra quant à lui en 1621 avec ses 330 passagers. La liste est longue de ces voyages qui furent les derniers. 
Certes certains êtres humains sont plus solides ou chanceux que d'autres, comme le met bien en évidence la biographie de l'infatigable Las Casas, qui sillonna les Antilles, l'Amérique centrale et le Mexique, réalisa un nombre impressionnant de voyages entre le Nouveau Monde et l'Espagne, et n'en vécut pas moins quatre-vingt-deux longues années.

Aux Philippines aussi, bien des hommes purent réaliser sans y succomber l'aller-retour entre l'archipel et la métropole. Parmi eux, bien sûr, des Espagnols, mais aussi des Asiatiques, notamment des vétérans originaires de la province de la Pampanga, au nord de Manille, qui avaient servi le roi d'Espagne dans les présides des Moluques. L'un d'entre eux, Nicolás de los Ángeles, avait pu se rendre durant sa jeunesse à la Nouvelle-Espagne où il avait obtenu du vice-roi le comte de Monterrey lui-même le droit de porter des armes et monter à cheval. Il revint ensuite aux Philippines avant de passer aux Moluques afin de servir sous les drapeaux du Monarque Catholique. À l'issue de sa carrière militaire, il quitta les Philippines en 1628 pour retourner au Mexique d'où il rédigea une relation de ses mérites qu'il allait présenter personnellement à la cour en 1631, pour s'en retourner ensuite vers la terre de ses pères, certainement en 1632.

Voilà donc un parcours, accompli uniquement afin de réaliser une démarche administrative, qui ne dura pas moins de quatre années fondamentalement consacrées à la réalisation du voyage lui-même A.G.I., Fil., 40, 11; A.G.I., Fil. 1, 248). Et encore, notre soldat eut la chance de voir sa demande rapidement satisfaite; Jerónimo de Lugay, lui aussi un vétéran pampango, dut résider deux ans à Madrid, de juin 1652 à mai 1654 au moins, afin de voir sa requête prise en compte (A.G.I., Fil., 4, 32 \& 36; A.G.I., Fil., 348, 4, f 119r-119v), pour assez peu de temps d'ailleurs, puisque, apparemment, le décret fut perdu et dut faire l'objet de l'envoi d'un duplicata au gouverneur des Philippines en 1661(A.G.I., Fil., 348, 4, fo298r-299r). Cette infortune est d'ailleurs toute relative si l'on considère que, pour sa part, Nicolas de los Ángeles ne reçut sa pension que durant deux années, ce qui l'obligea à rédiger pas moins de dix-sept demandes, de 1634 à 1646, afin que fût pris en compte ce que lui avait accordé Madrid, avant de voir sa pension enfin confirmée en 1652 (A.G.I., Fil. 43, 9) ${ }^{16}$.

Bien sûr, on pourrait multiplier à l'envie les exemples de vies où la distance qui séparait la colonie philippine de l'Espagne pesa de tout son poids chronologique. Bien sûr, il y a aussi des vies de marins. Cependant, il fut une fonction coloniale bien particulière qui fit de cet aller-retour une authentique vocation et dont la raison d'être fut justement de pallier le plus incommensurable des obstacles à la circulation des informations émises par les Philippines: leur difficile compréhension par les autorités métropolitaines. Pour ce faire, un individu, le

1619 août 1634,14 juillet 1635,9 octobre 1635,26 novembre 1635, 5 septembre 1637 , 13 septembre 1638, 30 septembre 1638, 21 février 1639, 13 novembre 1639, 30 mars 1640, 26 avril 1640, 11 août 1640, 8 octobre 1641, 20 août 1642, 16 septembre 1644, 16 février 1646, 30 juillet 1646 et 16 septembre 1652 pour la confirmation du Conseil des Indes. 
procurador general, était chargé d'assurer la représentation de la colonie auprès de la cour. La fonction fut inaugurée en 1583 avec Gabriel de Ribera (Gaudin 2017) et serait tout particulièrement importante au XVII ${ }^{e}$ siècle, dans le cadre des «incompréhensions» madrilènes quant aux activités commerciales, largement illicites, de la communauté marchande manillaise.

Hernando de los Ríos Coronel est certainement le plus connu d'entre ces hommes dévoués aux intérêts de ces lointains bouts de terre. Né en 1559, il décida de se rendre aux Philippines en 1586, à ses vingt-huit ans, un âge relativement tardif pour les candidats à l'aventure ultramarine au $\mathrm{XVI}^{\mathrm{e}}$ siècle. Il atteignit en 1588 l'archipel où il exerça tout d'abord l'office de capitaine, ce qui l'amena jusqu'au Cambodge et en Chine, ainsi qu'à réaliser une carte de l'île de Formose (Taiwan). Nommé procurador en 1605, il partit pour la métropole le 10 juillet pour finalement l'atteindre en octobre de l'année suivante. Il y demeurerait jusqu'en juin 1610, et ne rejoindrait Manille qu'au milieu de l'année 1611. Il repartit vers l'Espagne en août 1617 et y arriva sans doute à la fin de l'année 1618. Malgré qu'il demandât de rentrer aux Philippines en 1621, il y demeura jusqu'à sa mort, dont la date se situe entre 1622 et 1624 (Crossley 2011) ${ }^{17}$.

Comment penser une telle existence entre trois mondes autrement qu'en termes de sacerdoce, un terme qui se confirme d'ailleurs, puisque Ríos Coronel se fit ordonner prêtre à Séville, sur le retour de son premier voyage de procurador en Espagne? Lui arrivait-il de se demander ce qu'il se passait dans un de ses mondes pendant qu'il vivait dans un autre? Se posait-il la question: «quelle heure est-il là-bas?»(Gruzinski 2008).

La particularité du voyage vers et depuis les Philippines, et conséquemment ce qui fit la spécificité temporelle de l'archipel, c'est la Mar del Sur, le Pacifique. La Monarchie Catholique revendiqua la possession de ce Lago Español (Spate 1979) ${ }^{18}$, puisqu'il se situait dans sa partie du monde en vertu du traité de Tordesillas et qu'elle fut la première à le traverser, puis à y tracer une route, celle du galion de Manille, connu sous le nom de nao de China de l'autre côté de l'océan.

Observons-le donc, dans l'éblouissante carte qu'en fit Abraham Ortelius en 1589.

De prime abord, on contemple un vaste espace, étonnamment clos - ce qui facilite son appréhension - du fait de cette immense terre australe, largement supputée, qui en ferme toute la partie inférieure. Mais de ce qui se trouve dans cet espace, qu'en connaissait-on réellement à l'époque? Au Sud-Ouest, la Nouvelle-Guinée et les îles Salomon ne furent qu’à peine reconnues. Au Centre-

17 John Crossley, Hernando de los Ríos Coronel and the Spanish Philippines in the Golden Age, Ashgate, Farnham, Surrey, 2011.

18 Notons que c'est contre cette revendication monopolistique sur un océan qu'Hugo Grotius va rédiger son Mare Liberum et ainsi contribuer de façon décisive à l'élaboration du droit international. 
Est, les îles Galapagos furent quant à elles bien découvertes dès 1535, mais guère fréquentées depuis lors en 1589. Regardons plus attentivement:

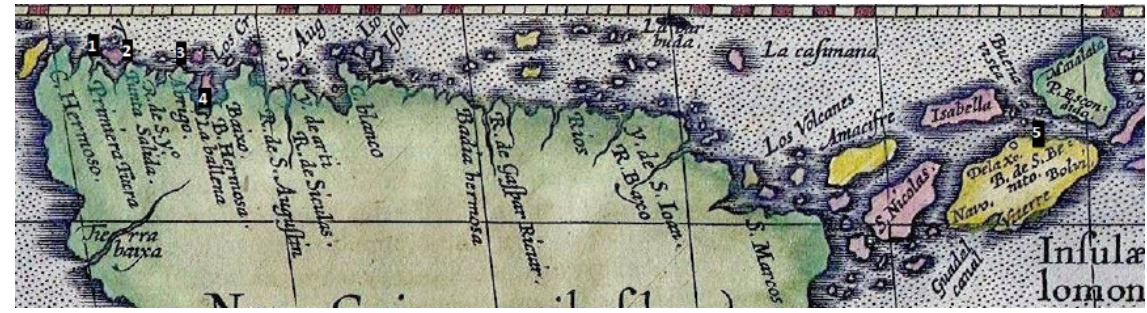

Abraham Ortelius, Maris Pacifi, 1585(détail). Carte reproduite avec l'aimable autorisation de Helmink Antique Maps.

Sur les côtes papoues, on peut lire parmi les toponymes: "Primera tierra »[1], «Punta Salida » [2], «Abrigo »[3]. Il s'agit là des étapes de l'itinéraire ayant permis la reconnaissance de ces terres, suite auxquelles, manifestement, les explorateurs rencontrèrent une baleine [4]. À gauche, au nord de l'île de Guadalcanal, de part et d'autre de la mention d'une «B[ahía] de S. Benito», on peut observer l'inscription "Dela Xo.» "Bolví » [5], qui apparaît aussi, cette fois de part et d'autre de l'île, dans la carte de Giovanni Mazza (1589) publiée la même année. Puisque " $\mathrm{X}^{\circ}$ » est censé désigner le Christ, elle pourrait signifier «je suis revenu de la croix», en référence à quelque péril vital s'étant présenté en cet endroit, mais il pourrait aussi s'agir de la déformation de la mention, dans le document ayant inspiré l'une et l'autre carte - très certainement le récit du premier voyage d'Álvaro de Mendaña (1567-1569) - d'un «debajo volví», «je suis revenu par en dessous». Dans les deux cas, il s'agirait à nouveau d'un témoignage de la façon dont le voyage à l'origine de ces données s'est déroulé.

Ainsi donc, nombre d'informations qui sont reportées dans cette réalisation, laquelle n'est pourtant pas une carte-portulan, sont largement virtuelles, puisque fondées sur un nombre limité d'expériences empiriques. Surtout, elles rendent finalement davantage compte d'une temporalité, celle des voyages qui permirent leur collecte, que de la spatialité de lieux véritablement parcourus. En somme, en 1589, si les terres asiatiques à l'Ouest et américaines à l'Est étaient bien des réalités spatiales, l'essentiel de la carte, ce qui lui donna son nom, existait essentiellement en creux. Or, ce creux, ce n'est pas de l'espace mais bien du temps, celui qui était nécessaire à la traversée de l'immense océan.

Mais que se passe-t-il pendant ce temps?

Bien sûr, on dispose d'éléments nous permettant d'entendre dans quelque mesure comment se déroulait le voyage: les piètres rations alimentaires, les effrayantes conditions d'hygiène, les préventions pour éviter les incendies, les jeux pour tuer le temps, les célébrations, et même la promiscuité de certains, d'autant plus coupable qu'en provoquant l'ire divine, elle mettait en péril tous 
les passagers ${ }^{19}$. Cependant, dans les lettres des administrateurs, seules quelques lignes rendent généralement compte de ces longs mois passés en mer. Les voyageurs sont certes plus prolixes dans leurs récits mais, dans la mesure où le «je» du début du XVII ${ }^{\mathrm{e}}$ siècle n'est pas encore un sujet au sens moderne du terme, ils ne s'attardent guère sur les interactions individuelles qu'ils ont pu tisser durant la traversée.

Pourtant, dans ce creux du Pacifique, dans ce temps suspendu dans leur chronologie vitale, les hommes échangeaient, sans doute même de façon intense, puisque l'intimité était absolument inévitable. De façon essentielle aussi puisque, d'Acapulco à Manille, ceux qui s'en retournaient dans un archipel dont ils avaient déjà l'expérience, ceux aussi et surtout qui s'apprêtaient à assumer des fonctions qui allaient construire la colonie dans le futur, se côtoyaient pendant trois mois. Ainsi, le dominicain Domingo de Salazar, le premier évêque des Philippines, et celui qui dirigeait le premier contingent de jésuites, Alonso Sánchez, arrivèrent aux Philippines par le même bateau. Une fois sur place, leur collaboration fut étroite, ce qui n'allait pas de soi entre des membres de ces deux congrégations. Qu'avait-il pu bien se nouer entre ces hommes de foi durant le voyage? Il y a là une sorte d'aîon, cet instant pur comme le conçoit Gilles Deleuze (1969: 195), un moment d'une immanente substantialité qui, inévitablement, nous échappe.

\section{L'espace du trop tard: le temps des occasions manquées}

\section{De fortuna \& infortuna}

Il existe une autre modalité de l'instant, toute aussi insaisissable que l'aîon, et qu'il convient pourtant aux hommes de saisir, lorsqu'ils le peuvent. S'en méfier surtout, car de cet instant bien souvent découlent de funestes conséquences pour celui qui ne l'a pas vu venir ou, tout au moins, pour celui qui n'a pas pris en compte la possibilité de son surgissement. C'est le kairos, ce coup de dés des choses que certains appellent destin, d'autres hasard, et qui, malgré eux, se joue dans la vie des hommes. Afin de parer à ses coups, Aristote recommande dans son Éthique à Nicomaque (VI, 5) de cultiver la phronesis, la prudence de celui qui sait qu'il ne saurait jamais se considérer comme totalement maître du jeu. À l'aube des temps modernes, Machiavel en appelle pour sa part à la virtù du Prince, qui se doit d'être capable de parer aux coups du sort ou au contraire, de faire fructifier la bonne fortune du moment afin de durer dans le temps (Rélang 2004). Pour Baltasar Gracián et les Espagnols de l'époque baroque qui s'agitent

19 Hernando de los Ríos Coronel, A.G.I., Filipinas, 27, 51, proposa ainsi dans un mémoire de 1605: «30: Qu'aucun des passagers ou marins n'amène avec lui des femmes esclaves car c'est une très grande offense faite à Dieu et on peut être sûr qu'elles sont perdues à l'arrivée à Acapulco. Il convient de mettre de l'ordre en cela car beaucoup ont commerce avec elles, si ce n'est les maîtres, d'autres passagers du navire et ce n'est pas raisonnable qu'en une circonstance où on prend tant de risques, il y ait occasion d'irriter Dieu et j'ose affirmer que depuis dix ans que cette pratique s'est établie, beaucoup de catastrophes sont survenues». 
sous nos yeux distants depuis leur lointaine colonie philippine, c'est l'ocasión (Soto Rivera 2006), ce moment qui parfois mena à la construction d'empires, parfois à leur chute, tantôt ce "presque rien» (Jankélévitch 1957), cette poussière dans le rouage de toute politique, qu'elle soit individuelle ou collective, tantôt cet éclair, ce coup de tonnerre qui peut tout aussi bien galvaniser que désespérer, mais dont il s'agit dans tous les cas de s'accommoder, et qu'idéalement, il faut parvenir à apprivoiser.

Un Hernán Cortés, aussi effroyable que furent les conséquences de sa virtù, sut indéniablement faire siennes une incroyable série de coïncidences qui, en 1519, constituèrent son occasion. Mais en 1527, lorsqu'il lança son cousin à la conquête des Moluques, le coup de dés, cette fois-là, ne donna pas la combinaison espérée.

En Asie, les Portugais jouirent d'une stupéfiante série d'occasions favorables, un alignement de planètes difficilement prévisible lorsque Vasco de Gama atteint en 1498 un port de Calicut où les marchands orientaux regardèrent d'abord avec dédain les piètres marchandises apportées par ces étrangers. Arrivés dans les eaux qui baignent l'Arabie peu avant que les Ottomans en contrôle les côtes, en Inde moins de trente ans avant la fondation de l'empire Moghol, les serviteurs de Manuel Ir pénétrèrent une Insulinde où les sultanats musulmans, déjà très dynamiques d'un point de vue commercial, n'étaient pas encore polarisés d'un point de vue politique et religieux. Ils découvrirent la Chine de l'Empire Ming à la veille de sa décadence, au moment idéal où celui-ci connaissait une grande prospérité économique tout en étant menacé au Nord par les Mongols, au Sud par les attaques des pirates wakō japonais, et commençait à se déliter, de sorte que la présence portugaise put sembler tout à la fois intéressante, et comparativement peu menaçante, et put compter sur la corruption des fonctionnaires locaux. Ils arrivèrent enfin au Japon à la fin de l'époque Sengoku, marquée par une atomisation politique qui leur permit de faire le jeu des seigneurs locaux, les daïmios, avides d'indépendance et de pouvoir sur leurs voisins, ce que semblait leur permettre le commerce économique et religieux avec ces barbares hirsutes. Les temps étaient ainsi particulièrement propices aux nouveaux-venus (Boxer 1948).

Mais bien vite, une après l'autre, les pièces qui constituaient le jeu de cette Asie lusitaine se retournèrent: les Ottomans lancèrent une série d'expéditions anti-portugaises et tissèrent des alliances avec tous ceux qui ruminaient contre ces intrus. En Inde, sous les règnes d'Akbar puis de Jahangir, les Moghols tissèrent un réseau de voies commerciales qui ne pouvaient qu'inquiéter. $\mathrm{Au}$ Japon, la réunification du pays impliqua un rejet des pernicieuses influences étrangères, que les Portugais représentaient en premier lieu. En Insulinde, leurs succès allaient susciter une belligérance anti-chrétienne que porterait tout particulièrement le sultanat d'Aceh. Dans le Golfe Persique, l'alliance des Safavides avec les anglais mena à la chute d'Ormuz, pivot de la présence portugaise dans la région. En Chine, l'effondrement de l'Empire Ming rendit la situation de 
Macao particulièrement instable. Enfin, partout, les néerlandais bousculèrent violemment les positions des Ibériques (Subrahmanyam 1999: 185-228).

Les Espagnols, pour leur part, s'établirent en Asie entre ces deux phases. Leur temporalité fut donc profondément différente, d'autant plus que leur présence en Extrême-Orient constitua une prolongation de leur expansion en Amérique, alors qu'il en fut à l'inverse pour les Portugais. En réalité, ils n'eurent guère qu'un peu moins de vingt ans, du début des années 1580 jusqu'en 1600, pour découvrir librement cet espace asiatique qu'ils avaient tant convoité, puisque, de 1565 jusqu'en 1580, date de l'union des Couronnes ibériques, l'illégalité de la présence des Espagnols dans l'archipel - puisque il est censé se trouver en zone portugaise - et la conséquente opposition des Lusitains, qui réalisèrent un siège maritime de leur position en 1568, interdit aux espagnols de réellement se projeter au-delà de l'archipel philippin. À partir de 1600, le péril néerlandais changea la donne et convertit l'Asie des Ibériques en une extension du conflit qui en Europe opposait l'Espagne à ses «rebelles flamands». L'Asie qu'ils entrevirent à travers cette mince fenêtre de deux décennies, du début des années 1580 jusqu'en 1600, n'était plus celle des jours glorieux des hommes de Manuel le Fortuné, et les occasions à saisir, assurément moins nombreuses.

Au-delà du champ des grands moments, il faut bien souligner un trait saillant de l'ocasión espagnole en ces lointaines terres d'Orient: la stupéfiante déveine des serviteurs du monarque catholique.

Le gouverneur Gómez Pérez Dasmariñas (1589-1593), qui fut chargé de diriger la flotte enfin capable de livrer les Moluques à l'Espagne. Le 17 octobre 1593, il quitta les eaux philippines à la tête d'une imposante armada constituée d'un galion, six galères et une myriade de voiles légères. Mais au deuxième jour de navigation, il fut assassiné dans la galère amirale au cours d'une mutinerie des rameurs chinois qui avaient été enrôlés de force. Fin de l'aventure. Le gouverneur Pedro Bravo de Acuña (1603-1606), qui parviendrait enfin à conquérir les Moluques en avril 1606, décéda brutalement quelques mois après son retour triomphal aux Philippines, sans doute empoisonné, sans avoir pu assurer la pérennisation de sa victoire. Le gouverneur Juan de Silva (1609-1616), qui fut justement l'homme à qui cette pérennisation et, plus largement l'expulsion des Néerlandais d'Asie, seraient confiées, quitta le port de Cavite le 31 décembre 1615 à la tête d'une prodigieuse flotte, «la plus grande armada qu'on ait vue dans ces îles, et certainement dans les Indes» d'après le jésuite Valerio de Ledesma (Pastells 1931: CCCXLVII-CCCL), qui ne comptait pas moins de 10 galions, 4 galères ainsi que d'autres embarcations légères, afin de rejoindre les forces portugaises à Malacca. Il y mourut le 1 avril 1616 de malaria et, avec lui, la tant attendue riposte décisive aux avancées des rebelles flamands. Il faut ajouter à ces décès spectaculaires le nom des gouverneurs qui succombèrent durant leur mandat: mentionnons ainsi Gonzalo Ronquillo (1580-1583), qui avait pourtant été nommé gouverneur à vie, et Alonso Fajardo de Tenza (1618-1624), qui trois 
ans plus tôt avait donné la mort à l'épouse infidèle et au soldat qui avec elle l'avait trahi (Calvo 2019) ${ }^{20}$, tous deux supposément morts d'une crise de mélancolie.

Les décès ne sont pas les seules infortunes qui, brusquement, ont le pouvoir d'abolir le temps ordinaire sur lequel on devait pouvoir compter. L'archipel philippin est en effet un des recoins de la planète les plus exposés aux catastrophes naturelles, situé qu'il est à la jonction de deux plaques tectoniques, sur la ceinture de feu du Pacifique et dans la trajectoire des typhons qui y naissent. Les catastrophes naturelles, forcément imprévisibles à l'époque, ou semi-naturelles, telles que les naufrages de navires, furent donc très fréquentes durant la période qui nous occupe. Ainsi, si l'on ne mentionne que la seule décennie de 1650, il est vrai particulièrement généreuse en termes de désastres impromptus, on dénombre, en sus des trois naufrages et deux départs avortés mentionnés plus haut, un tremblement de terre (1659) qui détruisit Manille, qu'on était encore en train de reconstruire suite au précédent (1645), et plusieurs dizaines de typhons qu'un examen attentif des textes de l'époque permettrait de documenter. On saisit ainsi à quel point Sabiniano Manrique de Lara (1653-1663), gouverneur des Philippines durant dix longues années de 1653 à 1663, qui dut aussi faire face à trois insurrections indigènes de grande ampleur, un soulèvement du quartier chinois de Manille et une menace d'invasion par le très puissant pirate Koxinga, eut certainement l'occasion de cultiver la circonspection face à l'incertitude des choses (Sanchez 2019).

Ces coups du sort, ces accidents du temps, furent d'autant plus funestes en vertu de la distance et de l'isolement de l'archipel puisque la réponse de Madrid pour pallier l'accident et donner les moyens aux Philippines de renouer avec le cours normal des choses était forcément longue à venir. Un coup brusque du temps qui fait encore davantage sentir sa longueur, qu'il étend, encore.

\section{Les Moluques, encore et toujours}

Au début de cet essai comme de l'aventure asiatique dont il se fait l'écho, les Moluques se sont imposées, comme au voyageur leur cônes volcaniques où s'accrochent les nuages. Lorsqu'on évoque le premier siècle de présence hispanique en Extrême-Orient, impossible de faire l'impasse sur elles. Pourtant, la route maritime aussi bien que l'itinéraire chronologique qui mènent vers ces îlots exigus constituèrent bel et bien une impasse. Tapies derrière les Philippines, tour à tour comme un désir tenace, une menace persistante ou un regret pérenne, dures, les Moluques durent.

Reconstituons donc le fil de l'amer destin moluquois de l'Espagne ${ }^{21}$.

Ni Magellan, qui mourut sur l'île de Mactan sous les flèches des hommes du vieux Lapu-lapu, ni Enrique, son esclave malais et peut-être l'homme qui

20 La légende veut que l'histoire du gouverneur des Philippines trompé aurait inspiré Pedro Calderón de la Barca pour sa pièce El médico de su honra.

21 Pour une synthèse des événements et des principaux enjeux de l'occupation espagnole des Moluques, on peut se référer à Jean-Noël Sánchez Pons, 2009. 
réalisa avant tous les autres le tour de la planète (Bertrand 2020), n'atteignirent ces minces bouts de terre volcanique où règne le giroflier. C'est ainsi à Elcano, le second de l'infortuné portugais, que revint cet insigne honneur en 1522. Deux autres expéditions partirent dans les années qui suivirent: celle de García Jofre de Loaisa, qui quitta La Corogne en 1525, et celle de Hernando de Saavedra Cerón, la première à lever l'ancre depuis les côtes du Mexique, en 1527. Mais si elles atteignirent bien, quoiqu'en piteux état, les précieuses Îles aux Épices, nul navire espagnol, à l'instar de la Victoria d'Elcano quelques années plus tôt, ne parvint alors à réaliser le voyage retour par la route Pacifique. De guerre lasse, l'empereur Charles Quint décida alors d'hypothéquer ses droits potentiels à revendiquer la possession des Moluques en faveur de son futur beau-père Jean III du Portugal; c'est le traité de Saragosse de 1529. Malgré tout, en 1541, une flotte commandée par Ruy López de Villalobos laissa derrière elle la Nouvelle-Espagne pour l'Asie avec l'accord tacite des autorités compétentes. Même si les Moluques restaient évidemment en ligne de mire, traité de Saragosse oblige, l'objectif n'était alors officiellement plus l'Épicerie mais bien les Philippines, qui seraient baptisées au cours de ce voyage du nom du prince héritier. C'est ainsi l'obscur désir espagnol d'effluves moluquoises qui donna aux Philippines leur première raison d'être et le fond de leur parfum.

Les Espagnols s'installeraient finalement aux Philippines en 1565. Pourtant, comme on vient de le voir, ils se garderont bien dans un premier temps de s'approcher de trop près de l'archipel du giroflier, jusqu'à ce que les circonstances européennes changent la donne à partir des années 1580. Dès lors, ce furent les Portugais, expulsés de Ternate, l'île principale des Moluques, en 1575, qui s'empressèrent d'humblement demander l'assistance de leurs nouveaux frères d'armes, lesquels lancèrent effectivement une série d'opération de reconquête des Moluques en 1582, 1583, 1585, sans succès. Viendrait ensuite l'ambitieuse expédition de 1593, tuée en mer avec le gouverneur Gómez Pérez Dasmariñas. Suivirent ensuite quelques années de divertissement, au sens pascalien du terme, marquées par l'illusion d'une conquête du Cambodge ${ }^{22}$.

Conséquemment, les Moluques, qui avaient suscitées le départ de Magellan en 1519, et qui ne cessèrent jamais de susciter convoitise et projets espagnols depuis lors n'étaient toujours pas terres du Roi Catholique à la fin du Xvi ${ }^{\mathrm{e}}$ siècle. Mais les temps avaient bien changé depuis l'année où Charles Ier d'Espagne devint empereur. Une force éminemment hostile s'apprêtait à faire une entrée fracassante dans le jeu asiatique des Ibériques: les rebelles néerlandais, en guerre contre leur roi Philippe II depuis 1568. Parvenus à Java en 1595, les bataves que l'on pensait d'abord d'inoffensifs marchands, attaquèrent Manille en 1600,

22 Cette tentative de conquête du Cambodge constitue justement un objet d'étude particulièrement intéressant eu égard à la notion de temporalité. Cependant, plutôt que d'y consacrer un nombre de lignes nécessairement insuffisant, nous avons préféré ici faire l'impasse sur cet épisode complexe. Il fera éventuellement l'objet d'un travail ultérieur. 
marquant ainsi brutalement le changement de siècle. L'heure n'était plus aux rêves d'aventures. Le temps, brusquement, accélérait. 1603: nouvelle tentative de conquête des Moluques par des forces luso-espagnoles, nouvel échec. 1606: une flotte constituée de 5 galions, 4 galères pour un total de 36 embarcations 1400 soldats espagnols 3095 hommes au total parvint enfin à réaliser la reconquête des Moluques.

Mais il était sans doute déjà trop tard.

L'Espagne n'avait plus l'initiative puisque, l'année précédente, ce furent les Hollandais qui d'abord, s'emparèrent des Îles aux Épices. Et puis on ne put guère penser encore longtemps que l'archipel avait été reconquis, car si le victorieux gouverneur Acuña - qui mourut quelques mois plus tard - put dire au lendemain de sa victoire (A.G.I., Patronato, 47, 7), qu'elle lui semblait un rêve ${ }^{23}$, c'est parce qu'il n'y avait eu aucune confrontation avec la flotte néerlandaise, qui mouillait à ce moment-là dans les eaux javanaises. Et le songe d'Acuña, au rythme effréné des copieux envois de navire depuis Amsterdam, devint très vite le cauchemar de ses successeurs. Dès la fin de la première décennie du $\mathrm{XVII}^{\mathrm{e}}$ siècle, les Espagnols avaient perdu l'essentiel du terrain au profit de leurs compétiteurs septentrionaux, assurément aussi forts sur mer qu'en diplomatie asiatique (Reid 1999).

Ce moment, décisif, amène à penser une autre temporalité et d'autres lenteurs structurelles, celles qui sont propres à l'appareil décisionnel madrilène à l'époque où le très corrompu duc de Lerma était aux rennes de la Monarchie de Philippe III. Si l'on se penche d'une part, sur les minutes des consultations du Conseil des Indes ${ }^{24}$, notamment concernant la question de la guerre, et d'autre part, sur les cédules royales émises par cette même institution, on constate qu'on envisageait l'envoi vers l'Asie d'une force navale massive dès le lendemain de la victoire en trompe-l'œil - ce que l'on ne savait pas encore en Espagne - de 1606 (A.G.I., Fil., 1, 118). On nomma à cet effet pour nouveau gouverneur des Philippines l'auguste Juan de Silva, brillant vétéran de la guerre des Flandres et chevalier de l'ordre de Saint-Jacques. Pressé d'en découdre - il faut dire que la VOC inaugura son entrée en fonction par un blocus de la baie de Manille de quatre mois et demi - celui-ci lança trop rapidement, et sans disposer pour cela ni de l'aval de la métropole, ni de celui des membres de l'audiencia manillaise, une première expédition vers les Moluques en 1611, sans aucun résultat probant. Pendant ce temps, à Madrid, la junta de guerra du Conseil des Indes se réunissait le 20 mai 1611 pour commenter la victoire obtenue par Silva à l'issue du siège naval de 1610. On lui envoya des félicitations en même temps qu'une copie des

23 «Parece cosa de sueño este suceso».

24 Pour ce faire, nous avons utilisé les volumes 1605-1609, 1610-1616 et 1617-1625 du Catálogo de las Consultas del Consejo de Indias, établis par Heredia Herrera (1983, 1984, 1985). Il est à noter que, pour les références qui ne sont pas issues de la section Filipinas, l'éditrice ne donne pas la référence exacte des pages du document, ce pour quoi nos propres références sont dans ce cas imprécises. 
conditions de la trêve de douze ans (1609-1621) négociée avec les ProvincesUnies afin qu'il la respectât (A.G.I., Fil., 2, 274). Sept mois plus tard, pourtant, on lui demandait de n'en rien faire (A.G.I., Indiferente, 1867).

Puis, silence en ce qui concerne les opérations militaires aux Philippines dans les ordres du jour des réunions du Conseil des Indes durant près de trois ans. Du côté des cédules royales, faisant suite à une série d'ordres données afin de mobiliser des forces portugaises pour les Philippines, le 10 mars 1613, on informait Juan de Silva de l'envoi de 400 soldats et de six vaisseaux qui seraient acheminées via la route lusitaine du cap de Bonne Espérance (A.G.I., Fil., 329, $\left.2, \mathrm{f}^{\circ} 167 \mathrm{v}-168 \mathrm{v}\right)$.

Le 9 octobre 1614, le Conseil considéra l'envoi d'un secours substantiel aux Philippines. Mais le texte de la consulta manifestait un flou des plus inquiétants quant à l'origine des moyens nécessaires à l'opération: Espagne? Inde portugaise? Mexique? Philippines? La tête madrilène ne semblait pas savoir quelle partie du corps de la monarchie solliciter pour aller de l'avant (A.G.I., México, 2487). Un an plus tard, la junta n'avait toujours pas trouvé de réponse satisfaisante à cette question à choix multiples. Puis, à nouveau les Moluques disparaissent des ordres du jour du Conseil des Indes jusqu'au début de l'année 1617.

On a déjà évoqué dans la deuxième partie de cet essai la cédule royale du 15 décembre 1614 qui demandait des nouvelles des îles. Mais la grande surprise vient de ce qui suit, ou plutôt de ce qui ne suit pas: aucune cédule sur la guerre en Asie durant toute l'année 1615 et, plus généralement - du moins d'après ce que nous avons pu vérifier - seulement trois, sur des questions peu transcendantes, adressées à Manille contre 39 en 1614 et 124 en $1616^{25}$.

Au début de l'année 1616, la machine s'emballa. Le 25 janvier 1616, on écrivit au Capitaine Général des hommes de guerre du Portugal pour lui demander de réunir des renforts destinés aux Philippines (A.G.I., Fil., 329, 2, f $187 \mathrm{v}-188 \mathrm{v}$ ). Le 5 février, on fit la même demande au duc de Medina Sidonia, dans une cédule à la fin de laquelle on peut lire:

Idem au Corregidor de Cadix, Idem au Marquis de Ayamonte et sa province, Idem au Corregidor de Gibraltar, Idem au Corregidor de Malaga, Idem au Marquis de Villanueva del Fresno en ce qui concerne le port de Máguez et sa région, Idem au Duc de Peñaranda en ce qui concerne le port de Palos et sa région, Idem au Duc de Medina Celi en ce qui concerne El Puerto de Santa María. (A.G.I., Fil., 329, 2, f ${ }^{\circ}$ 189r)

Le 28 mars, le Conseil écrivit à l'audiencia de Manille afin qu'elle prît la direction des opérations au cas où le gouverneur venait à mourir (A.G.I., Fil., 329, 2, f $193 \mathrm{v}-194 \mathrm{v})$, ce qui, surviendrait en effet une vingtaine de jours plus tard.

Juan de Silva n'était donc plus, et avec lui l'impressionnante armada, fruit d'une colossale mise à contribution de la colonie faute de renforts suffisants

25 Décompte effectué sur la base, de la liste des consultas, censée être exhaustive, réalisée par Heredia Herrera (1984). 
envoyés par Madrid. Mais le Conseil des Indes ne le savait pas, et il espérait encore parvenir à envoyer les forces enfin capables de mettre un terme à la présence hollandaise en Asie. Le 30 décembre 1616, on annonça ainsi au futur gouverneur Alonso Fajardo de Tenza que l'armada initialement destinée aux Philippines avait été finalement déroutée pour une opération dans le détroit de Gibraltar, mais on continuait à penser à la «nécessité que parte cette année le secours pour les Philippines», étant donnée "l'impossibilité qu'il y aura pour l'année prochaine» (A.G.I., Fil., 329, 2, fo 238v-239r).

Les consultas du Conseil, encore et encore, se suivirent, sans autre conséquence que de laisser le dossier moluquois ouvert sur la table des affaires urgentes. L'historien en vient ainsi à esquisser un sourire lorsqu'il peut lire sous la plume du président Carrillo, en date du 25 août 1619: "Je vous avise qu'il y a ici des lettres de Séville qu'on suppose d'une personne avisée, dans lesquelles il est dit que cet envoi est très en retard et qu'il est à craindre qu'il ne puisse partir dans les temps nécessaires» (A.G.I., Mex., 2487).

Deux ans plus tard, le constat serait sans appel:

Il faut bien considérer ce qu'ont coûté des armadas des Philippines sans qu'elles aient eu d'effet du fait qu'on n'a pas mesuré le temps qui était nécessaire pour leur préparation et envoi. (A.G.I., Mex., 2487)

Ne pas avoir "mesuré le temps qui était nécessaire», et fatalement, en avoir perdu. En 1619, l'année où, déjà éreintée, l'Espagne rajoutait sur ses épaules un prodigieux fardeau militaire en entrant dans la Guerre de Trente Ans, il était vraisemblablement déjà trop tard pour espérer gagner la partie. Et pourtant, elle devait encore rester aux Moluques pendant de nombreuses années, au prix de la mobilisation de centaines de soldats indigènes philippins, espagnols ou mexicains, de l'angoisse constante de l'envoi de ravitaillements en péril d'être attaqués par les flottes ennemies, au prix surtout de plus de 200000 pesos annuels, soit l'essentiel du déficit de la colonie philippine. Elle resterait jusqu'en 1648, date de la signature du traité de Munster qui mit un terme à la guerre de Quatre Vingt Ans avec les Provinces-Unies et du traité de Westphalie qui marqua la fin de la Guerre de Trente Ans, et avec elle des illusoires rêves impériaux de la Monarchie Catholique. Elle resta même au-delà, jusqu'en 1663, alors qu'il n'y avait plus rien à gagner, plus personne à combattre.

Lorsqu'on démantela le préside espagnol de Ternate, la Couronne sembla alors se réveiller d'une léthargie de près d'un demi-siècle et demanda qu'on lui expliquât la raison du retrait des forces espagnoles de ces îles qui lui avaient tant coûté. On lui répondit unanimement que la présence hispanique n'y avait eu aucun sens, ni militaire, puisque l'Espagne y perdait surtout la réputation de ses armes, ni économique, puisque les Bataves récoltaient l'essentiel de la précieuse girofle, ni religieux, car les autochtones n'avaient jamais été très enclins à 
embrasser le christianisme ${ }^{26}$, autant d'arguments qui avaient déjà été proposés par le jésuite Francisco Combés (1654).

Pourquoi donc être resté si longtemps?

Aux Philippines, certainement du fait d'une multitude de petites et grandes convenances. En effet, à côté de l'urgence militaire, le début du XVII ${ }^{\mathrm{e}}$ siècle fut aussi une période de formidable ébullition autour du commerce de produit chinois exportés vers les Amériques. Dans ce contexte, Macao, la sœur jumelle tout à la fois aimée et détestée, était la partenaire indispensable des grandes opérations commerciales interlopes. Or, ces amours tumultueuses ne purent officiellement plus être avec la déclaration d'indépendance du Portugal en 1640. Dès lors, les Moluques purent constituer un formidable alibi pour que les frères d'hier devenus ennemis pussent continuer leurs affaires plus au Sud, au port de Macassar, à l'extrémité de la branche Ouest de l'île des Célèbes, fréquenté depuis longtemps par les Portugais et où les Espagnols stationnés aux Moluques s'approvisionnaient depuis les années 1610. Macassar, c'était aussi un sultanat tardif - ses souverains n'embrassèrent l'islam qu'autour de 1605 - qui se développa tout particulièrement au moment même où la VOC commençait à imposer dans la violence le monopole dont elle entendait jouir sur la production et la vente des épices de la région. Macassar, qui tint tête aux forces de l'implacable Jan Peterszoon Coen, s'affirma alors comme le dernier port franc de l'est de l'Insulinde. Ainsi, le maintien aux Moluques permit aux espagnols de prolonger le temps luso-espagnol en Asie après que celui-ci eut cessé officiellement d'être. Le sultanat offrit aussi un havre où les perdants de la compétition coloniale en Insulinde pouvaient encore continuer à jouer ensemble, même après qu'il ne fut trop tard, jusqu'à ce qu'ils ne le pussent plus : les Néerlandais se décidèrent en 1660 à employer les grands moyens pour faire plier le port rebelle qui tomba définitivement entre les mains bataves en 1669 (Sánchez Pons 2020).

Pourquoi donc être resté si longtemps?

À Madrid, sans doute en partie par négligence, par habitude, voire par oubli. Mais peut-être encore, aussi paradoxal que cela puisse paraître, peut-on voir autre chose dans cette prolongation du temps des Moluques au-delà de sa fin. Du fait même que le songe moluquois s'était changé si tardivement en réalité, il était peut-être devenu en quelque sorte partie intégrante de ce plus vaste rêve que constituait le fantasme espagnol de Monarchie Universelle. Or, puisque Philippe II avait continué la politique impériale de son père sans en avoir le titre et donc la légitimité, comment son successeur, en 1598, pouvait-il changer de cap? Comment acter un changement d'orientation politique radical et, ce faisant, donner à voir aux yeux du monde, que tout cela n'avait été que fausse route, illusion et perte de temps? Il fallait donc continuer, jusqu'au fatal dénouement, comme Don Quichotte, le valeureux chevalier errant, dut refuser jusqu'au bout

26 Le dossier du démantèlement des positions espagnoles aux Moluques se trouve dans A.G.I., Filipinas, 201, 1. 
d'admettre qu'il n'était qu'Alonso Quijano, vieux gentilhomme désargenté, sous peine de mourir. Il en fut sans doute de même en quelque manière pour les Îles aux Épices, où, pour justifier le cap initial du rêve asiatique de l'Espagne, il avait fallu, par point d'honneur, par entêtement, que l'Espagne, par l'entremise de ses gouverneurs, persévérât contre vents et marées, contre l'implacable réalité d'une époque qui n'était clairement plus celle du rêve initial. Pour que le temps, passé, en vain, ne soit pas juste et seulement ce que, en fin de compte, il fut: du temps perdu.

\section{Conclusion}

$\mathrm{Au}$ sein de la grande famille des langues austronésiennes, les idiomes philippins sont réputés posséder un système de conjugaison complexe dont la difficulté est redoublée par la curieuse tendance de leurs locuteurs à affectionner tout particulièrement le mode passif. Pourtant, on peut tout à fait s'exprimer en tagalog ou en bisaya (cebuano) sans n'avoir rien à conjuguer, tout simplement en utilisant deux mots: $p a$, qui signifie encore, et $n a$, qui signifie déjà. On peut leur ajouter une négation, suivant les cas wala, que les cebuanophones aiment à réduire en $w a$, et dili, réductible en $d i^{27}$. Avec deux mots et deux syllabes, on peut ainsi exprimer toutes les modalités du temps.

L’Espagne avait déjà eu son "déjà " $(n a)$ en Amérique, pour construire une Asie espagnole qui n'était «toujours pas» (wala $p a)$. Une fois conquise, celle-ci serait inexorablement soumise à une inexorable attente structurelle, faisant d'elle l'espace de l' «encore» $(p a)$ qui, à bien des égards, et en particulier eu égard à ses projets moluquois, était aussi celui du «déjà plus» (wala na).

Mais quel est le sens d'user de mots des autochtones pour décrire la geste de ceux qui les asservirent et, justement, parlèrent à leur place? Car, il convient de le reconnaître, le travail qui ici se clôt n'a guère laissé d'espace à l'histoire de ceux qu'on a appelé à leur place philippins ${ }^{28}$.

En cette fin d'itinéraire, que dire donc de leur temps?

Assurément, l'inclusion dans le temps chrétien constitua un considérable bouleversement pour les habitants de l'archipel: c'est le temps computable du calendrier grégorien, mais aussi le temps suspendu au-dessus du temps des grands mythes du christianisme, qui marqua profondément les pratiques

27 En cebuano, wala, littéralement «rien» nie une possession, comme dans «wala'y panahon", "il n'y a plus de temps", ou constitue la négation d'un verbe conjugué au passé, tandis que «dili» nie une existence ( dili na sundalo»: «il n'est plus soldat»), un état ( dili na buhi»: «il n'est plus vivant») un auxiliaire modal («dili na mahimo ( pwede»: "cela n'est plus possible») ou un verbe conjugué au présent ou au futur. Pour se familiariser avec la structure du cebuano, nous recommandons Trosdal (1992).

28 En réalité, les Espagnols réservaient le terme de Filipino aux créoles espagnols nés dans l'archipel. Ce furent justement les ilustrados qui s'approprièrent le gentilé pour désigner les autochtones. 
autochtones du dernier pays où, aujourd'hui, on pratique encore des crucifixions à clous réels afin de réactualiser dans le présent la passion que jadis endura le fils de Dieu.

Hors du temps, c'est bien là où la "monacocratie» dénoncée par les intellectuels philippins de la fin du $\mathrm{xIX}^{\mathrm{e}}$ siècle maintenaient ceux que les missionnaires appelaient encore Indios tandis que les jeunes Rizal, Del Pilar, López Jaena et Luna, qui avaient vécu en Europe, rêvaient de changements véloces. Mais si les revendications de cette élite hispanophone constituèrent un des chemins qui menèrent à la révolution qui éclata en 1895, il en fut un autre, plus sinueux.

Au sein des masses paysannes, notamment tagalogs, l'idée d'un rejet légitime de l'autorité espagnole fut progressivement formulée en termes d'annulation de la dette morale des autochtones envers l'Espagne, tandis que le parcours des autochtones était identifié aux souffrances christiques. Cette assimilation se fit notamment à travers la diffusion d'écrits de dévotion sur la Passion rédigés en langues locales et par le biais de la réappropriation locale de la figure de Bernardo del Carpio, héros de nombreux romances médiévaux péninsulaires, sorte de Roland espagnol fort mal récompensé pour ses exploits militaires au service des rois des Asturies. L'awit (long poème versifié) qui lui est consacré en tagalog, l'Historia Famosa ni Bernardo Carpio, publiée en 1860 et largement diffusée oralement dans les années qui suivirent, induirait l'assimilation de l'Espagne au souverain ingrat et des Philippines à un Bernardo Carpio autochtone qui serait enfermé dans les montagnes de Montalban, au nord-est de la région de Manille, d'où il s'apprêterait à sortir pour libérer le peuple (Ileto 1979). Ainsi, le temps révolutionnaire, qui en tant que sursaut de nature millénariste constitue toujours une abolition du temps, découla en partie, comme pour les Comedias de Moros y cristianos devenues moro-moro, d'une resémantisation d'un récit issu d'un lointain passé hispanique.

Vint donc l'insurrection, puis la guerre hispano-américaine, la mise en place d'un nouveau cycle colonial. Mais laissons-là l'histoire et consacrons les mots de la fin au présent.

Ce qui est assurément fascinant dans l'idiosyncrasie philippine, c'est la coexistence de strates culturelles, la malaise (et la chinoise pour de nombreux individus héritiers d'un métissage interasiatique), l'hispanique ${ }^{29}$ et la nordaméricaine, lesquelles cohabitent dans le présent malgré qu'elles correspondent en quelque manière à différentes temporalités. Et, puisque de temps il est question, il est difficile de ne pas évoquer cette stupéfiante capacité qu’ont les

29 Il faudrait d'ailleurs employer hispanique au pluriel, puisque, dans le culte aux saints par exemple, on peut clairement distinguer ce qui tient de l'héritage mexicain (le culte à la vierge de Guadalupe, patronne du pays par exemple) prioritaire jusqu'au début du XIX ${ }^{\mathrm{e}}$ siècle, et ce qui fut importé ultérieurement d'Espagne après les indépendances hispano-américaines (les cultes ruraux péninsulaires comme celui consacré à San Isidro Labrador). 
individus à le tuer, dans une attente bonhomme, tout particulièrement dans ces jolis kiosques en bambou présents au bord des routes, dans chaque puro ${ }^{30}$, où on discute, joue, mange et boit en attendant l'heure où il faudra bien faire autre chose. Dans la langue populaire, on appelle d'ailleurs ces lieux de convivialité des tambayan, une acclimatation malicieuse du terme anglais stand-by, celui qu'on trouve sur les boutons qui permettent de mettre les machines en veille.

Mais les Philippines ne sont pas hors du temps, loin de là. Dans une large mesure au contraire, elles sont au plus haut point prises et modelées par le temps d'un monde globalisé qui est le nôtre. Près du quart de tous les marins, de ceux qui permettent aux produits que nous consommons quotidiennement d'arriver jusqu'à nous, sont ainsi philippins. Plus généralement, plus de 10 millions de Philippins vivent à l'étranger pour y travailler, ce qui fait du pays le plus important pourvoyeur de main d'œuvre de la planète (C.F.O. 2014). La majorité de ces individus sont des femmes, employées dans le secteur de l'aide aux personnes (domestiques, gardes d'enfants, infirmières), qui ont souvent laissé au pays leur propre progéniture, confiée à des proches auxquels on envoie de l'argent, "hinay hinay, basta kanunay", "doucement doucement, mais constamment», comme on a coutume de dire en cebuano. Parfois, des années passent avant que les familles ne puissent être à nouveau réunies, pour un temps seulement, une vacance entre deux contrats (Parreñas 2001).

Aujourd'hui, aux Philippines, comme au XVII ${ }^{\mathrm{e}}$ siècle, on attend ainsi souvent l'arrivée de l'aide envoyée d'un lointain ailleurs, et la flotte qui, on l'espère, par la mer ou plus fréquemment par les airs, ramènera avec elle ceux qui trop longtemps sont partis.

\section{Bibliographie}

Albert-Llorca M. \& González Alcantud J. A., 2003, (éds), Moros y Cristianos, representaciones del otro en las fiestas del Mediterráneo Occidental, Toulouse, Presses Universitaires du Mirail.

Archivo General de Indias: Filipinas, 1, 118 \& 248 / 2, 274; 4, 32 \& 36 / 8, 1, 5 / $18^{\mathrm{a}}, 2,9 / 20,9,56 / 27,51 \& 63 / 40,11 / 43,9 / 63,1 / 201,1 / 329,2, \mathrm{f}^{\circ} 167 \mathrm{~V}$ $168 \mathrm{v} ; 185 \mathrm{r} ; 187 \mathrm{v}-188 \mathrm{v} ; 189 \mathrm{r} ; 193 \mathrm{v}-194 \mathrm{v} ; 238 \mathrm{v}-239 \mathrm{r} / 330,4$, f $155 \mathrm{v}-156 \mathrm{r} /$ 340, 3, fo 266r-267r / 348, 4, fo 119r-119v / 348, 4, fo298r-299r. Indiferente, 1867. México, 2487 / Patronato, 47, 7.

Aristote, 1994, Éthique à Nicomaque, Paris, Vrin.

Bernand C. \& Gruzinski S., 1991, Histoire du Nouveau Monde, De la découverte à la conquête, 1492-1550, Paris, Fayard.

30 L'équivalent du barrio hispanique est aux Philippines le barangay, lui-même divisé en purok. 
Bertrand R., 2020, «Et si Enrique avait été le premier?», L'Histoire, n476, p. 50-55.

Bigot Morogues, P.M.S., 1812, Mémoire historique et physique sur les chutes des pierres tombées sur la surface de la terre à diverses époques, Orléans, Imprimerie de Jacob ainé.

Boxer C., 1948, Fidalgos in the Far East, 1550-1570, La Haye, Martinus Nijhoff.

Cáceres Valderrama M., 2005, La fiesta de moros y cristianos en el Perú, Lima, Pontificia Universidad Católica.

Calvo T., 2019, Espadas y Plumas en la Monarquía Hispana, Madrid, Casa de Velázquez.

Center for Filipinos Overseas (CFO), 2014, Compendium of Statistics on International Migration 2013, Manille, Office of the President, Commission on Filipino Overseas.

Chirino P., 1604, Relación de las islas filipinas, Rome, Esteban Paulino.

Chaunu P., 1969, Conquête et exploitation des nouveaux mondes, Paris, PUF «Nouvelle Clio», $\mathrm{n}^{\circ} 26$ bis.

Collectif, 1840, Presupuestos de gastos e ingresos de las islas de Cuba, Puerto-Rico y Filipinas para el año 1839, Madrid, Imprenta Nacional.

Colín F., 1663, Labor evangelica, ministerios apostolicos de los obreros de la Compañia de Iesvs, fvndacion, y progressos de su provincia en las islas Filipinas, Madrid, José Fernández de Buendía.

Combes F., 1654, Relación destas Islas filipinas dividida en tres partes y un Discurso Político de las Malucas (manuscrit), Biblioteca de Palacio, Ms. II/3062.

Corpuz O., 1997, An economic history of the Philippines, Quezon City, University of the Philippines Press.

Crossley J., 2011, Hernando de los Ríos Coronel and the Spanish Philippines in the Golden Age, Farnham, Ashgate.

Deleuze G., 1969, Logique du sens, Paris, Les éditions de minuit.

Del Vas Mingo M.M., 1985, «Las Ordenanzas de 1573, sus antecedentes y consecuencias», Quinto Centenario 8, Complutense de Madrid, p. 83-101.

Domínguez García J., 2006, «Santiago mataindios: la continuación de un discurso medieval en la Nueva España», Nueva Revista de Filología Hispánica, 54, n 1, p. 33-56.

Donoso I., 2010, “The Hispanic moros y cristianos and the Philippine komedya”, Philippine Humanities Review, 11-12, 2009-2010, p. 87-120.

García Abásolo A., 2000, «Formación de las Índias Orientales Españolas. Filipinas en el siglo XVI», in Cabrero L. (éd.), Historia General de Filipinas, Madrid, Ediciones de Cultura Hispánica, p. 171-205.

Gracián B., 1651, El Criticón, Primera parte, Saragosse, Juan Nogues.

Gaudin G., 2013, Penser et gouverner le nouveau monde au XVII siècle: L'empire de papier de Juan Diez de la Calle, Paris, L'Harmattan. 
Gaudin G., 2017, «Un acercamiento a las figuras de agentes de negocios y procuradores de Indias en la Corte», Nuevo Mundo Mundos Nuevos, Debates, en ligne.

Gil J., 2011, Los chinos en Manila, siglos XVI y XVII, Lisbonne, Centro Científico e Cultural de Macau.

Gruzinski S., 2004, Les Quatre Parties Du Monde, Histoire d'une mondialisation, Édition de la Martinière.

Gruzinski S., 2008, Quelle heure est-il là-bas? Amérique et islam à l'orée des temps modernes, Paris, Seuil.

Gutiérrez L., 1992, Historia de la Iglesia en Filipinas, Madrid, editorial MAPFRE.

Hanke L., 1943, Cuerpo de documentos del siglo XVI sobre los derechos de España en las Indias y las Filipinas, Mexico, Fondo de Cultura económica.

Heredia Herrera A., 1983-1984-1985, Catálogo de las Consultas del Consejo de Indias, établis par Antonia Heredia Herrera (ed.), Séville, Diputación provincial de Sevilla.

Ileto R., 1979, Pasyon and Revolution, Popular Movements in the Philippines, 1840-1910, Manille, Ateneo de Manila University Press.

Jankélévitch V., 1957, Le je-ne-sais-quoi et le presque-rien, Paris, PUF.

Las Casas B. de, 1551, Brevísima relación de la destrucción de las Indias, Séville.

Mazza, G. B., c. 1589, Americae et Proximar Regionum Orae Descriptio, Venise, Donati Rascicotti formis.

Morineau M., 1978, «Les mancenilliers d'Europe», in Léon P. (dir.), Histoire économique et sociale du monde, 2, Paris, Armand Colin, p. 139-162.

Ortelius A., 1589, Maris Pacifici, Anvers, Christophe Plantin.

Parreñas R., 2001, Servants of Globalization: Women, Migration and Domestic Work, Stanford University Press.

Pastells P., Historia General de Filipinas, in Navas del Valle F., 1931, Catálogo de los documentos relativos a las islas Filipinas existentes en el Archivo General de Indias de Sevilla, VI, Barcelone, Compañía General de Tabacos de Filipinas.

Phelan J., 1959, The Hispanization of the Philippines, Madison, The University of Wisconsin Press.

Pilar M. del, 1889, La frailocracia filipina, Barcelone, Imprenta Ibérica de Francisco Fossas.

Prieto Lucena A.M., 1984, Filipinas durante el gobierno de Manrique de Lara, 1653-1663, Séville, Escuela de Estudios Hispano-Americanos de Sevilla.

Real Academia de la Historia, 1889, Boletín de la Real Academia de la Historia, Tome 15, 1889.

Reid A., 1999, "Early Southeast Asians Categorizations of European », in Reid A., Charting the shape of early modern Southeast Asia (recueil d'articles de l'auteur), Bangkok, Silkworm Books, p. 155-180.

Rélang A., 2004, "La dialectique de la fortune et de la virtù chez Machiavel», Archives de Philosophie, 66, 2003/4, p. 649-662. 
Retana W., 1909, Noticias histórico-bibliográficas de El teatro en Filipinas desde sus orígenes hasta 1898, Madrid, Librería General de Victoriano Suárez.

Ribadeneyra P. de, 1595, Tratado de la religión y virtudes que debe tener el príncipe cristiano para gobernar y conservar sus Estados. Madrid, Emprenta de P. Madrigal.

Ricard R., 1932, "Contribution à l'étude des fêtes de "moros y cristianos" au Mexique», Journal de la Société des Américanistes, 24, n. 1, p. 51-84.

Rivero Rodríguez M. \& Gaudin G. (coords.), 2020, "Que aya virrey en aquel reyno" Vencer la distancia en el imperio español, Madrid, Polifemo.

Sanchez J.N., 2017, "La construction discursive du Moro philippin aux Xvi et $\mathrm{XVII}^{\mathrm{e}}$ siècles», in Jacquelard C. \& BénatTachot L. (dir.), E Spania, 28, La place de l'Asie dans l'historiographie de la monarchie catholique, en ligne.

Sanchez J.N., 2019, "A Prismatic Glance at one Century of Threats on the Philippine Colony», Crailsheim E. (éd.), External Threat in World History, Leyde, Brill, p. 343-365.

Sánchez Pons J.N., 2009, «Tiempos Malucos. España y sus Islas de las Especias, 1565-1663», in Truchuelo García S. (dir.), Andrés de Urdaneta: un hombre moderno, Lasarte-Oria, Ayuntamiento de Ordizia, p. 621-650.

Sánchez Pons J. N., 2020, «Tardíos amores insulindios: Manila y el sultanato de Macasar en el siglo XVII», Vegueta, 20, p. 295-325.

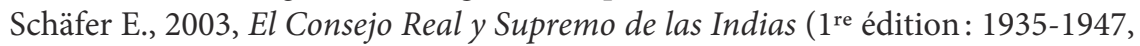
Séville, Imprenta M. Carmona) Madrid, Marcial Pons Historia.

Schurz W., 1939, The Manila Galleon, New York, Dutton \& Co.

Solorzano J. de \& Pinelo L., 1681, Recopilación de las Leyes de los Reynos de las Indias, Madrid.

Soto Rivera R., 2006, "El concepto de Ocasión en Baltasar Gracián», Conceptos. Revista de Investigación Graciana, 3, p. 69-84.

Spate O., 1979, The Spanish Lake, Canberra, ANU Press.

Subrahmanyam S., 1999, L'Empire portugais d'Asie, 1500-1700 (1 $1^{\text {re }}$ édition: 1993, The Portuguese Empire in Asia 1500-1700, Londres, Longman) Paris, Maisonneuve et Larose.

Trosdal M., 1992, Formal-Functional Grammar of the Cebuano Language, Cebu, Salvador \& Pilar Sala Foundation.

Videira Pires B., 1987, A viajem de comércio Macau-Manila nos séculos XVI a XIX, Macao, Centro de Estudos Marítimos de Macau.

Vitoria F. de, De Indis, 1532, in Relectiones theologicae, Lyon, Jacobum Boyer, 1557. 